\title{
Trois profils-types de jeunes affichant des problèmes de comportement sérieux
}

\section{Three Type Profiles in young people with serious behavour problems}

\section{Tres perfiles-tipo de jóvenes que presentan serios problemas de comportamiento}

\author{
Marie-Hélène Gagné, Nadia Desbiens et Katherine Blouin
}

Volume 32, numéro 1, printemps 2004

La violence en milieu scolaire

URI : https://id.erudit.org/iderudit/1079127ar

DOI : https://doi.org/10.7202/1079127ar

\section{Aller au sommaire du numéro}

\section{Éditeur(s)}

Association canadienne d'éducation de langue française

ISSN

0849-1089 (imprimé)

1916-8659 (numérique)

Découvrir la revue

Citer cet article

Gagné, M.-H., Desbiens, N. \& Blouin, K. (2004). Trois profils-types de jeunes affichant des problèmes de comportement sérieux. Éducation et francophonie, 32(1), 276-311. https://doi.org/10.7202/1079127ar
Résumé de l'article

Les conduites violentes des jeunes font partie d'un ensemble de comportements inadaptés qui prennent généralement racine dans des contextes familiaux et sociaux difficiles. Une compréhension juste du développement des conduites violentes chez les jeunes doit tenir compte de ce portrait global. Cet article rapporte les résultats d'une recherche qualitative ayant pour but de cerner la spécificité des profils psychosociaux et des besoins des jeunes qui, tout en affichant des problèmes de comportement sérieux, présentent également un vécu de mauvais traitements physiques, psychologiques ou sexuels à l'intérieur de leur famille. Les résultats proviennent de l'analyse exhaustive des dossiers cliniques de 12 garçons et 11 filles de 9 à 17 ans recevant des services de protection de la jeunesse. Ces 23 dossiers ont été dépouillés afin de documenter l'évolution simultanée des problèmes de comportement, des pratiques parentales maltraitantes, du contexte familial, du contexte scolaire et de l'intervention de protection (signalements, évaluations, mesures appliquées, placements). L'analyse qualitative, effectuée de manière à respecter la chronologie de la trajectoire de vie de chaque jeune, a permis de dégager trois profils-types de jeunes à la fois victimes de maltraitance et éprouvant des problèmes de comportement sérieux : l'Indésirable, l'Explosif et le Délinquant. Chacun de ces profils est décrit et mis en perspective à l'aide des théories de l'attachement, du trauma et de la socialisation, respectivement, ce qui permet de proposer au milieu scolaire des pistes d'intervention différentielles pour ces différents types de jeunes en difficulté.
Tous droits réservés @ Association canadienne d'éducation de langue française, 2004
Ce document est protégé par la loi sur le droit d'auteur. L'utilisation des services d'Érudit (y compris la reproduction) est assujettie à sa politique d'utilisation que vous pouvez consulter en ligne. 


\title{
Trois profils-types de jeunes affichant des problèmes de comportement sérieux
}

\author{
Marie-Hélène Gagné \\ École de psychologie, Université Laval, Canada
}

Nadia Desbiens

Faculté d'éducation, Université de Montréal, Canada

\section{Katherine Blouin}

Faculté des sciences de l'éducation, Université Laval, Canada

\section{RÉSUMÉ}

Les conduites violentes des jeunes font partie d'un ensemble de comportements inadaptés qui prennent généralement racine dans des contextes familiaux et sociaux difficiles. Une compréhension juste du développement des conduites violentes chez les jeunes doit tenir compte de ce portrait global. Cet article rapporte les résultats d'une recherche qualitative ayant pour but de cerner la spécificité des profils psychosociaux et des besoins des jeunes qui, tout en affichant des problèmes de comportement sérieux, présentent également un vécu de mauvais traitements physiques, psychologiques ou sexuels à l'intérieur de leur famille. Les résultats proviennent de l'analyse exhaustive des dossiers cliniques de 12 garçons et 11 filles de 9 à 17 ans recevant des services de protection de la jeunesse. Ces 23 dossiers ont été dépouillés afin de documenter l'évolution simultanée des problèmes de comportement, des pratiques parentales maltraitantes, du contexte familial, du contexte scolaire et de l'intervention de protection (signalements, évaluations, mesures appliquées, place- 
ments). L'analyse qualitative, effectuée de manière à respecter la chronologie de la trajectoire de vie de chaque jeune, a permis de dégager trois profils-types de jeunes à la fois victimes de maltraitance et éprouvant des problèmes de comportement sérieux: l'Indésirable, l'Explosif et le Délinquant. Chacun de ces profils est décrit et mis en perspective à l'aide des théories de l'attachement, du trauma et de la socialisation, respectivement, ce qui permet de proposer au milieu scolaire des pistes d'intervention différentielles pour ces différents types de jeunes en difficulté.

\section{ABSTRACT}

Three Type Profiles in young people with serious behavour problems

Marie-Hélène Gagné, Laval University

Nadia Desbiens, University of Montréal

Katherine Blouin, Laval University

Violent behaviour in young people is one part of a number of maladaptive behaviours, usually originating from difficult family and social contexts. To understand the development of violent behaviour among youth, we must look at the big picture. This article reports the results of a qualitative study whose goal was to define the specificity of psychosocial profiles and the needs of students with serious behaviour problems, who also experienced physical, psychological or sexual abuse within their families. An exhaustive analysis was made of the clinical files of 12 boys and 11 girls who received youth protection services, ranging in age from 9 to 17 years old. The 23 files were studied in order to document the simultaneous evolution of behaviour problems, abusive parental practices, the family context, the school context and youth protection interventions (reporting cases, evaluations, measures taken, placements). The qualitative analysis, done respecting the chronology of the life path of each child, brought to light three type profiles of children who were both victims of abuse and had serious behaviour problems: The Undesirable, The Explosive and The Delinquent. Each of these types is described and put in perspective in relation to attachment theories, trauma and socialization, respectively, allowing the suggestion of differential intervention paths to the school milieu for these different types of students in difficulty. 
RESUMEN

\section{Tres perfiles-tipo de jóvenes que presentan serios problemas} de comportamiento

Marie-Hélène Gagné, Universidad Laval

Nadia Desbiens, Universidad de Montreal

Katherine Blouin, Universidad Laval

Los comportamientos violentos de los jóvenes forman parte de un conjunto de comportamientos inadaptados que se enraízan generalmente en contextos familiares y sociales difíciles. La comprensión justa del desarrollo de conductas violentas entre los jóvenes debe tomar en consideración esta visón global. Este artículo reporta los resultados de una investigación cualitativa que tenía como objetivo la identificación específica de los perfiles psico-sociales y las necesidades de los jóvenes que presentan serios problemas de comportamiento, pero que también han vivido malos tratos físicos, sicológicos o sexuales al interior de sus familias. Los resultados provienen del análisis exhaustivo del dossier clínico de 12 muchachos y 11 muchachas de 9 a 17 años que beneficiaron de los servicios de protección de la juventud. Los 23 dossier fueron analizados con el fin de documentar la evolución simultanea de los problemas de comportamiento, de las prácticas paternas mal-tratantes, del contexto familiar, del contexto escolar y de la intervención de protección (denuncias, evaluaciones, medidas aplicadas, internamientos). El análisis cualitativo, efectuado para respectar la cronología de la trayectoria de vida de cada joven, permitió identificar tres perfiles-tipo de jóvenes víctimas de maltrato y que presentan serios problemas de comportamiento: el Indeseable, el Explosivo y el Delincuente. Describimos cada uno de esos perfiles, situándolos en perspectiva gracias a las teorías del apego, del trauma y de la socialización, respectivamente, lo que permite proponer al medio escolar algunas pistas de intervención diferenciales para los diferentes tipos de jóvenes con dificultades.

On estime qu'entre $5 \%$ et $10 \%$ des jeunes de 8 à 16 ans de pays industrialisés présentent des troubles persistants du comportement.

\section{Introduction}

Les difficultés d'adaptation sociale touchent un nombre important de jeunes. On estime qu'entre $5 \%$ et $10 \%$ des jeunes de 8 à 16 ans de pays industrialisés présentent des troubles persistants du comportement (Hill, 2002). Par conséquent, il n'est pas surprenant que la violence en milieu scolaire soit fréquente, voire normative (Craig, Peters, \& Kornaski, 1998) et mondialisée (Debarbieux, 2003). Au Canada par exemple, une enquête menée auprès d'un échantillon représentatif de 6259 enfants de $6^{\mathrm{e}}, 8^{\mathrm{e}}$ et $10^{\mathrm{e}}$ années révèle que $13,6 \%$ des jeunes rapportent avoir usé d'intimidation envers d'autres jeunes, alors que 14,5\% disent en avoir été victime au long d'un trimestre (King, Boyce, \& King, 1999). 
L'étiologie des problèmes de comportement, incluant les conduites violentes, met en cause des facteurs de risque individuels, familiaux et sociaux qui interagissent au fil du développement selon des mécanismes complexes et encore mal connus (Hill, 2002; Reppucci, Fried, \& Schmidt, 2002). Certes, l'école a peu de prise sur certains de ces facteurs et mécanismes, notamment ceux qui touchent l'adversité familiale. Par contre, elle peut faire contrepoids en fournissant un modèle de climat relationnel sain, en instaurant des programmes de prévention de la violence et de promotion des habiletés sociales et en fournissant des services compensatoires aux jeunes en difficulté en collaboration avec d'autres organismes de la communauté (Bowen, Desbiens, Martin \& Hamel, 2001; Deslandes, 2001; Garbarino, 1987). L'école peut également contribuer à la prévention de la maltraitance envers les enfants. Par le biais de sa mission sociale d'éducation, l'école peut stimuler la responsabilité sociale des citoyens vis-à-vis des enfants et des jeunes; par le biais de ses pratiques institutionnelles, elle peut promouvoir une idéologie positive de l'enfant (Hart, 1988).

Toutefois, toute forme d'intervention pour contrer la violence en milieu scolaire doit se fonder sur une compréhension juste et globale de l'origine des conduites inadaptées et violentes des jeunes et de la manière dont elles se développent, notamment au sein du milieu familial. La présente étude contribue à cerner la spécificité des profils psychosociaux des jeunes qui, tout en affichant des problèmes de comportement sérieux, présentent également une histoire de maltraitance, afin de poser les fondements à une intervention différenciée en fonction de leurs besoins spécifiques.

\section{Recension des écrits}

Les enfants et les jeunes sont à risque de subir de nombreuses formes de victimisation (Finkelhor \& Dziuba-Leatherman, 1994). D'un autre côté, une proportion non négligeable de jeunes, en majorité des adolescents de sexe masculin, affiche des problèmes de comportement qui peuvent s'exprimer par des gestes agressifs ou violents (Farrington \& Loeber, 2000). À l'aide de données provenant de diverses sources, D’Amours (1995) a bien illustré ces "deux côtés de la médaille de la violence " chez les jeunes. Cette métaphore convient puisque les deux problématiques seraient liées : les enfants victimes de violence, d'abus ${ }^{1}$ et de négligence sont plus susceptibles que les autres d'afficher des problèmes d'agressivité (Erickson \& Egeland, 1987; McGee, Wolfe \& Wilson, 1997; Vissing, Straus, Gelles, \& Harrop, 1991). Après avoir effectué une recension des écrits empiriques sur ces liens, Jonson-Reid (1998) propose un modèle où la maltraitance et l'exposition à la violence conjugale, entre autres, devraient être étudiés comme des facteurs qui contribuent à la violence des jeunes.

Il est largement reconnu que la violence et la négligence parentale, l'abus sexuel et l'exposition à la violence conjugale ont des impacts délétères majeurs sur le développement des enfants (Cicchetti \& Toth, 1995; Kolbo, Blakely, \& Engleman, 1996; Trickett \& Putnam, 1998). Les jeunes enfants maltraités développeraient

1. Dans ce document, le terme "abus » et ses dérivés est utilisé dans un sens typiquement nord-américain. Il désigne des formes sévères, ou abusives, de violence commises à l'endroit de mineurs, excluant la négligence. 
notamment des problèmes d'attachement et de régulation émotionnelle, ce qui les amènerait à éprouver des difficultés dans leurs relations interpersonnelles, puis dans leur adaptation scolaire. Ces impacts seraient particulièrement graves lorsque différentes formes de victimisation se juxtaposent l'une à l'autre au fil du développement ou lorsqu'elles s'inscrivent dans des trajectoires d'expériences répétées (Carlson, 1991; Crittenden, Claussen \& Sugarman, 1994; Higgins \& McCabe, 2000; McGee et al., 1997; O’Keefe, 1995; Vissing et al.,1991). Or, cette juxtaposition semble constituer la règle plutôt que l'exception chez les jeunes qui proviennent de milieux familiaux et sociaux plus détériorés (Claussen \& Crittenden, 1991; Crittenden et al., 1994; Erickson \& Egeland, 1987; McGee et al., 1997; Vissing et al., 1991). Bref, les enfants sévèrement maltraités risquent de vivre un enchaînement d'événements négatifs qui les place à haut risque de s'engager dans une trajectoire d'échecs répétés, de marginalisation et d'inadaptation, incluant l'apparition de problèmes de comportement. Une telle trajectoire est préoccupante puisque les problèmes de comportement qui débutent dès l'enfance sont reconnus comme les plus stables dans le temps, pouvant persister jusqu'à l'âge adulte, ce qui laisse entrevoir leur caractère

Des études longitudinales démontrent que les jeunes qui manifestent des problèmes de comportement sont particulièrement susceptibles de présenter des tendances suicidaires sérieuses, de faire usage de drogues, de fuguer de

la maison, de vivre une grossesse précoce ou d'abandonner l'école. réfractaire à l'intervention (Dobkin, Tremblay, Mâsse \& Vitaro, 1993; Dryfoos, 1990) .

Le développement des problèmes de comportement s'opérerait graduellement et la famille peut être vue comme le premier lieu d'entraînement aux conduites antisociales (Dishion, French \& Patterson, 1995; Reid, Patterson, \& Snyder, 2002). Dans un milieu familial dysfonctionnel sur le plan relationnel et disciplinaire, l'enfant peut développer des conduites aversives et coercitives et apprendre à exercer un contrôle sur les autres. À son entrée à l'école, ce type de comportement risque de provoquer des conflits avec les pairs et se traduire par des problèmes d'opposition à l'autorité, ce qui peut déboucher sur le rejet social et l'échec scolaire. En retour, ces jeunes risquent de s'associer à des groupes de pairs déviants qui encouragent les comportements antisociaux et la délinquance (Patterson, Reid, \& Dishion, 1992) et en venir à présenter une constellation de problèmes d'adaptation et d'insertion. En effet, des études longitudinales démontrent que les jeunes qui manifestent des problèmes de comportement sont particulièrement susceptibles de présenter des tendances suicidaires sérieuses (Tousignant, 1993), de faire usage de drogues (Hawkins, Catalano \& Miller, 1992), de fuguer de la maison (Kennedy, 1991), de vivre une grossesse précoce (Forget, Bilodeau \& Tétreault, 1992) ou d'abandonner l'école (Rumberger, 1995).

Somme toute, tant la violence subie que la violence agie constituent des menaces au développement et à la sécurité des jeunes et contribuent de façon synergique à leur marginalisation et leur exclusion sociale. Ce constat justifie que l'on se préoccupe systématiquement des deux côtés de la médaille de la violence chez les jeunes en difficulté. Les jeunes filles et garçons qui reçoivent des services de protection comptent sans doute parmi les plus touchés par cette double problématique.

L'Étude d'Incidence Québécoise (ÉIQ) sur les signalements en protection de la jeunesse (Tourigny, Mayer, et al., 2002) jette un éclairage inédit sur cette clientèle. Effectuée dans 16 des 19 centres jeunesse du Québec, l'ÉIQ a permis de documenter 9790 signalements aux services de protection dont la moitié ont été retenus pour évaluation. Dans l'ensemble, 39 \% des jeunes signalés afficheraient des problèmes de 
comportement sérieux ${ }^{2}$, soit environ 11 jeunes sur 1000 dans la population générale des 0-17 ans. Il s'agit du second motif de signalement le plus fréquent après la négligence parentale ( $43 \%$ ), mais le principal motif pour les garçons de tous âges et pour les adolescent-e-s. Par ailleurs, le tiers des signalements retenus impliquent une double problématique. Les problèmes de comportement sérieux se juxtaposent à la négligence dans $10 \%$ des cas, aux mauvais traitements psychologiques dans $4 \%$ des cas, à l'abus physique dans $3 \%$ des cas et à d'autres formes de menaces au développement, incluant l'abus sexuel, dans $3 \%$ des cas. On peut même croire que si l'on avait considéré l'histoire antérieure de ces jeunes en matière de signalements et de mauvais traitements, les liens entre les problèmes de comportement et la victimisation auraient été encore plus probants.

De façon générale, cette recension d'écrits montre que la victimisation et les

La victimisation et les problèmes de comportement sont deux problématiques interdépendantes, toutes deux susceptibles de constituer les pierres d'assise de nombreuses difficultés ultérieures. problèmes de comportement sont deux problématiques interdépendantes, toutes deux susceptibles de constituer les pierres d'assise de nombreuses difficultés ultérieures. Les jeunes qui présentent à la fois ces deux problématiques comptent sans aucun doute parmi les individus qui présentent les plus grands besoins dans notre société, non seulement d'être encadrés et protégés, mais également d'être soulagés du traumatisme de la violence (Harris \& Fallot, 2001). Sur le plan des pratiques, on peut toutefois se demander jusqu'à quel point nos interventions sont suffisamment sensibles aux trajectoires de développement et aux dynamiques sous-jacentes à cette double problématique et, par conséquent, différenciées en fonction des besoins spécifiques de ces jeunes.

Dans un tel contexte, il apparaît essentiel de consentir des efforts pour mettre ces trajectoires, dynamiques et besoins spécifiques en relief. La présente étude, exploratoire et qualitative, se centre sur les jeunes qui affichent des problèmes de comportement et qui ont également eu à souffrir de diverses formes de maltraitance (abus et négligence de nature psychologique, physique ou sexuelle dans leur milieu familial). La recherche qualitative permet «l'analyse holistique de phénomènes complexes, dynamiques et exceptionnels " (Camic, Rhodes, \& Yardley, 2003, p. 9), ce qui correspond bien à l'objectif de la présente étude. Son but ultime consiste à sensibiliser et à outiller tant les intervenants sociaux, les intervenants en réadaptation que les enseignants afin de les aider à développer une meilleure compréhension des jeunes en difficulté et de leurs besoins, de faciliter l'évaluation des signalements et l'orientation de ces jeunes au sein du réseau des services sociaux et de fournir des pistes pour l'intervention et le suivi de ces jeunes.

2. Les principaux troubles de comportement sérieux renvoient aux problèmes de relation avec les parents ou avec l'autorité, aux problèmes de la conduite en milieu scolaire, à la consommation abusive d'alcool ou de drogue, à la violence verbale et physique envers les autres et aux comportements autodestructeurs ou de prise de risque. Sur le plan scolaire, ils incluent également l'absentéisme délibéré, les suspensions et les expulsions. 


\section{Méthodologie}

\section{Échantillon}

\section{Caractéristiques des participants}

L'échantillon qualitatif compte 23 jeunes, 12 garçons et 11 filles âgé-es entre 9 et 17 ans, recevant des services de protection du Centre jeunesse de Québec (CJQ), l'établissement qui chapeaute les services sociaux aux jeunes en difficulté et à leurs familles pour l'ensemble de la région administrative de Québec. L'âge moyen des filles est de 15 ans et celui des garçons est de 14,5 ans. Environ les trois quarts des jeunes ont vécu la séparation de leurs parents et trois jeunes ont perdu soit leur père, soit leur mère, dont deux par suicide. Seulement trois participant-e-s vivaient dans leur famille naturelle au moment de l'étude, les autres étant placés en famille d'accueil $(\underline{n}=6)$, en foyer de groupe $(\underline{n}=2)$ ou en centre de réadaptation $(\underline{n}=12)$. À l'école, ces jeunes présentent un profil global soit d'absence de motivation ou d'intérêt, soit d'opposition à l'autorité et de non respect des règles, souvent les deux à la fois, et enfin d'échecs et de redoublements pour la majorité d'entre eux. Ces données illustrent la grande vulnérabilité des jeunes de l'échantillon sur le plan familial et scolaire.

\section{Stratégie d'échantillonnage}

Ces jeunes ont été sélectionnés avec l'aide d'intervenants sociaux du CJQ à partir des critères suivants : (1) fille ou garçon âgé-e entre 10 et 17 ans; (2) les problèmes de comportement sont le principal motif de protection ou la majeure sur le plan de l'intervention; (3) présence d'une histoire de mauvais traitements. Ces situations de maltraitance pouvaient ou non avoir fait l'objet de signalements ou de mesures de protection dans le passé. L'important était que l'intervenant possède suffisamment d'information factuelle pour pouvoir certifier les mauvais traitements, incluant: (a) violence ou abus physique; (b) négligence des besoins physiques de base; (c) violence ou abus psychologique; (d) négligence des besoins affectifs de base; (e) exposition à la violence conjugale; (f) abus sexuel.

La stratégie d'échantillonnage privilégiée correspond à ce que Pirès (1997) nomme "échantillon par homogénéisation », permettant l'étude exhaustive et approfondie d'un groupe restreint dont les membres affichent des caractéristiques semblables. L'obtention d'une diversité au niveau de l'âge et du genre a également guidé l'échantillonnage. Une telle «diversification interne » favorise l'atteinte de la saturation empirique (Mucchielli, 1996; Pirès, 1997), un critère-clé de la validité du savoir produit en recherche qualitative. Plus la saturation empirique est atteinte, plus les résultats sont transférables, i.e. valides même lorsque transposés dans d'autres contextes. Ainsi, parmi les 35 dossiers qui ont été référés aux responsables de la recherche, 23 ont été sélectionnés pour respecter ces exigences méthodologiques. 


\section{Sources de données, instruments et procédures}

Moyennant une entente de confidentialité avec l'établissement, deux sources d'information ont été consultées : (1) le dossier de protection des jeunes référés et (2) les registres informatisés du CJQ ${ }^{3}$. Les documents sont traditionnellement reconnus comme une source de données en recherche qualitative, au même titre que les entretiens et les observations (Wolcott, 1992, cité dans Miles et Huberman, 1994, 2003).

\section{Dossiers de protection}

Les dossiers de protection ont été dépouillés à l'aide d'une grille préalablement conçue à cet effet et fondée sur une recension préliminaire des écrits. Afin de documenter et de classifier les problèmes de comportement, la typologie de Kauffman (1997) a été utilisée. Cette typologie comprend l'hyperactivité et les problèmes associés (impulsivité, inattention, manque d'autocontrôle) $(87 \%)^{4}$, les troubles ouverts de la conduite (agressivité, hostilité, « taxage »5 intimidation, agression sexuelle) (83\%), les troubles couverts de la conduite (vols, mensonges, opposition, problèmes scolaires, fugues) (100\%), la délinquance juvénile et l'usage de drogues (57\%), les troubles liés à l'anxiété et à l'isolement (introversion, troubles alimentaires ou sexuels) (70\%), la dépression et comportement suicidaire (78\%) et les comportements psychotiques (comportements extrêmement déviants, automutilation) (17\%). Quant aux mauvais traitements, ils ont été identifiés et classifiés à l'aide du Maltreatment Classification System (Barnett, Manly \& Chicchetti, 1993), une nosologie clinique développée à partir de cas de protection et fournissant des critères pour identifier les mauvais traitements " émotionnels " (incluant violence psychologique et négligence affective) (96\%), l'abus physique (52\%), l'abus sexuel (17\%), la négligence sous forme de défaut de soins ( $78 \%$ ) et de manque de supervision (48\%) et le mauvais traitement "moral-légal-éducationnel» (exposer le jeune à des modèles déviants, permettre ou encourager les conduites déviantes) $(70 \%)$.

Enfin, la grille a permis de documenter certains facteurs reconnus comme des déterminants de la santé et du bien-être des jeunes et pour lesquels les dossiers sont susceptibles de contenir de l'information. Sur le plan familial, on a documenté l'âge des parents, leur principale source de revenus, la composition familiale, l'histoire de séparation(s), recomposition(s) et formule(s) de garde de l'enfant, les problèmes présentés par les parents biologiques et les beaux-parents (alcoolisme, toxicomanie, santé mentale, déficience, violence conjugale, criminalité, prostitution et travail du

3. En vertu d'une disposition légale, les données sur un jeune sont conservées pour une durée maximale de cinq ans suite à la fermeture de son dossier. Concrètement, cela signifie que lorsqu'un dossier est fermé à la fin de I'application d'une mesure de protection et que le jeune en cause ne fait l'objet d'aucun signalement au cours des cinq années subséquentes, toutes les informations à propos de ce jeune sont susceptibles d'avoir été détruites. Il s'agit d'une limite inhérente à la source de données privilégiée pour cette étude.

4. Les pourcentages apparaissant entre parenthèses révèlent la proportion des jeunes de l'échantillon chez qui la dimension en question a été identifiée, tous niveaux de sévérité confondus. Pour l'abus physique et sexuel, les proportions représentent uniquement les cas fondés et excluent les cas où on notait un doute de l'intervenante.

5. Au Canada français, le terme « taxage » désigne un type particulier d'intimidation où un jeune subit différentes formes d'extorsion (argent de poche, vêtements, etc.) sous la menace d'un pair ou d'un groupe de pairs. 
sexe, tentatives de suicide, incarcération et hospitalisation). Sur le plan scolaire ${ }^{6}$, on a documenté le comportement du jeune, son rendement académique et son éventuel retard scolaire.

Avant d'en arriver à sa version finale, la grille d'analyse a été soumise à deux intervenant-e-s du CJQ afin de tester sa pertinence et son exhaustivité. Elle a également été prétestée sur deux dossiers, qui n'ont pas été inclus dans le corpus de données. Ensuite, les 23 dossiers sélectionnés ont été dépouillés à l'aide de cette grille par trois assistantes de recherche. La consigne consistait à prendre connaissance de l'ensemble du dossier, des documents et rapports officiels aux notes manuscrites des intervenant-e-s, puis à consigner toutes les informations requises par la grille d'analyse en les datant aussi précisément que possible. Par souci d'objectivité, la consigne exigeait aussi de noter uniquement l'information factuelle et d'exclure tout ce qui avait trait aux impressions et interprétations cliniques des intervenant-e-s.

Dans le but de maximiser la validité des données recueillies, sept des 23 dossiers ont été analysés indépendamment par deux analystes qui devaient ensuite s'entendre sur l'information à retenir dans un esprit de consensus. Cette procédure s'est effectuée sur les quatre premiers dossiers, puis à tous les cinq dossiers environ, de façon à assurer l'uniformité du début à la fin du processus de recueil des données.

\section{Les registres informatisés}

Les registres informatisés de l'établissement ont permis de documenter, pour chaque jeune, l'histoire des signalements (dates, motifs, rétention), les conclusions des évaluations (allégations fondées ou non, développement ou sécurité compromis ou non), les mesures de protection appliquées (dates, durée, services offerts) et les placements (dates, durées, type de ressource).

\section{Stratégie d'analyse qualitative}

À partir de l'ensemble de l'information recueillie, une histoire de cas a été rédigée pour chaque jeune de l'échantillon. En plus de permettre l'organisation des données à l'intérieur d'un même canevas, cette procédure a permis de reconstruire la chronologie de l'enchaînement des événements et des situations de vie qui ont accompagné le développement des problèmes de comportement chez chaque jeune, à partir de sa date de naissance puis à intervalles réguliers d'un an. Tous les antécédents parentaux et familiaux susceptibles d'avoir affecté la trajectoire de développement du jeune ont été consignés en prélude à chaque histoire de cas.

Ces histoires de cas ont ensuite donné lieu à une analyse séquentielle de synthèse: "Ce procédé revient à réaliser un "regroupement" de parcours individuels, même s'il est clair que lorsque l'on regarde de près, chaque carrière [ici, histoire de vie] est qualitativement distincte.» (Miles et Huberman, 2003, p. 363). Suivant un processus inductif, on a mis en parallèle les différentes histoires de cas afin de faire ressortir les similarités et les différences entre elles. Diverses techniques matricielles proposées

6. L'information obtenue sur le vécu scolaire du jeune est nécessairement parcellaire, compte tenu de la source d'information utilisée. Elle est tributaire de ce que les intervenant-e-s en protection de la jeunesse ont cru bon de consigner dans le dossier de protection et n'est donc pas uniforme d'un dossier à l'autre. 
par Miles et Huberman (2003) ont été utilisées pour croiser entre eux différents éléments des histoires de cas, de façon à dégager graduellement des profils-types de jeunes en difficulté.

Pour chacun des profils en développement et suivant trois grandes périodes de développement, soit la période préscolaire ( $0-5$ ans), l'âge scolaire (6-11 ans) et l'adolescence (12-17 ans), on a construit une matrice illustrant les signalements et les placements en milieu substitut, l'évolution de la structure familiale, les manifestations de maltraitance et leur sévérité approximative ${ }^{7}$, les manifestations de problèmes de comportement, les problèmes présentés par les parents, le vécu scolaire et enfin toute autre remarque jugée essentielle. Cette opération a permis de confirmer la cohérence et la stabilité des constellations de caractéristiques préalablement identifiées et de valider la typologie présentée ici.

\section{Résultats et discussion}

Le processus d'analyse a permis de dégager trois profils-types parmi les jeunes de l'échantillon, intitulés l'Indésirable, l'Explosifet le Délinquant en référence à leurs caractéristiques spécifiques. Dans les pages qui suivent, chacun de ces profils est décrit et interprété à la lumière d'une perspective théorique du développement, soit respectivement les théories de l'attachement, du trauma et de la socialisation.

\section{L'Indésirable}

Ce profil s'applique particulièrement à cinq jeunes de l'échantillon, trois garçons et deux filles. Il est caractérisé par des relations parents-enfant pauvres ou inexistantes dès la petite enfance. De façon générale, les parents sont jeunes et ont plusieurs enfants. Ils sont typiquement dépressifs, suicidaires, non disponibles ou non impliqués dans la vie de leur bébé et de leur jeune enfant, ce qui mène à la négligence, au rejet et même à l'abandon. Les mauvais traitements psychologiques sont une constante de ce profil, de la petite enfance à l'adolescence. Typiquement, ces jeunes sont signalés tôt aux services de protection, parfois dès leur naissance. Si certains connaissent des placements dès la petite enfance, tous vivent de nombreux placements à l'âge scolaire. Ces jeunes se montrent immatures, dépendants et exigeants vis-à-vis de l'adulte et affichent beaucoup de comportements de recherche d'attention. Ils ont tendance à être très agités et un trouble déficitaire de l'attention et d'hyperactivité (TDAH) est souvent diagnostiqué. Ils tendent à reproduire une dynamique de rejet dans divers contextes et à faire l'objet de rejet social de la part des pairs et des adultes.

7. Le Maltreatment Classification System (Barnett et al., 1993) fournit une échelle en cinq points pour estimer la sévérité de chaque forme de mauvais traitement. Les analystes se sont inspirées de cette échelle pour estimer la sévérité relative d'un cas par rapport à un autre. Toutefois, aucun indice d'accord inter-juges n'a été calculé sur les cotes de sévérité; c'est pourquoi les résultats rapportent la sévérité des situations de maltraitance de façon très globale, à titre indicatif seulement, lorsque c'est utile pour caractériser un profiltype en particulier. 
Du point de vue de la dynamique du fonctionnement familial, deux sous-profils peuvent être identifiés. Dans le premier cas, on retrouve un enfant «bouc émissaire » qui, par les caractéristiques peu désirables qu'il présente souvent (prématurité, malformation, maladie, déficience, tempérament difficile, comportement extrême, enfant non désiré), deviendra la cible de négligence affective, d'hostilité et de rejet explicite croissants de la part de ses parents. Cette dynamique relationnelle est susceptible d'exacerber les problèmes du jeune et de se chroniciser. Par ailleurs, les parents peuvent jouer adéquatement leur rôle avec leurs autres enfants. Ils peuvent collaborer avec les intervenants dans la mesure où ils conservent l'espoir de voir leur enfant "changer" (c'est lui qui a un problème), mais ont tendance à désinvestir lorsqu'ils constatent que ses problèmes de comportement demeurent ou s'intensifient. Ils tendent à nier leurs propres attitudes de rejet envers leur enfant. Ce sous-profil correspond assez bien à celui du «Vilain petit canard» identifié par Gagné et Bouchard (sous presse) dans une étude antérieure, ce qui appuie sa validité.

Dans le second sous-profil, on retrouve des mères qui ont un passé très lourd, incluant des expériences de mauvais traitements, de placements, de toxicomanie, de criminalité et de grossesses précoces, alors que les pères proviennent souvent d'un milieu semblable. Elles ont généralement eu leurs enfants très tôt, dans un contexte incompatible avec le rôle de parent. Les enfants se retrouvent avec des parents qui n'ont ni les capacités, ni la disponibilité pour s'occuper d'eux adéquatement, menant à de la négligence grave ou même à un abandon. Généralement, la situation des parents ne s'améliore pas vraiment avec le temps, ce qui amène pour le jeune une alternance de placements et de tentatives de réintégration échouées dans leur famille et à voir leurs espoirs déçus plus souvent qu'autrement.

Dans les deux cas, on aura affaire à des adolescents souffrants, opposants, non collaborateurs, renfermés et souvent suicidaires. Chez les enfants du second sousprofil en particulier, des comportements illicites ou délinquants s'observent également. Les tableaux 1, 2 et 3 résument les trajectoires de ces jeunes sur les plans de l'évolution de la famille et de l'intervention de protection, des mauvais traitements subis et des problèmes de comportement prédominants. Les caractères gras indiquent les caractéristiques spécifiques à l'Indésirable.

\section{Liens avec la théorie de l'attachement}

La théorie de l'attachement fournit un cadre explicatif intéressant aux problèmes de comportement de l'Indésirable. Les enfants maltraités, notamment ceux qui sont négligés, démontreraient un attachement à leur mère, ou à leur principal pourvoyeur de soins, significativement moins sécurisant que les enfants non maltraités (Benda \& Corwyn, 2002; Cole-Detke \& Kobak, 1998; Crittenden \& Claussen, 2000; Finzi, Cohen, Sapir, \& Weizman, 2000; Morton \& Browne, 1998; Wenar \& Kerig, 2000). De plus, les enfants dont l'attachement à leur mère est insécurisant seraient davantage à risque de développer des problèmes de comportement intériorisés ou extériorisés (Benda \& Corwyn, 2002; Cicchetti, Rogosh \& Toth, 1998; Cooper, Shaver \& Collins, 1998; Finzi \& al., 2000; Moss, Rousseau, Parent, St-Laurent, \& Saintonge 1998; 
Moss, St-Laurent, Rousseau, Parent, Gosselin, \& Saintonge, 1999; Pierrehumbert, Miljkovitch, Plancherel, Halfon, \& Ansermet, 2000).

Certaines caractéristiques typiques de l'Indésirable ont été associées à un style d'attachement insécurisant. La tendance à se faire rejeter par les autres et à se retirer socialement a été reliée au style d'attachement anxieux-ambivalent (Finzi et al., 2000). Benda et Corwyn (2002) ont observé que des mauvais traitements subis très tôt dans l'enfance, combinés avec un attachement faible, peuvent mener à des tendances suicidaires si l'enfant intériorise ses émotions, ou à la violence s'il les extériorise. Enfin, la dépression maternelle, vécue par plusieurs Indésirables de notre échantillon, est vue comme une menace pour la création du lien d'attachement (Cicchetti et al., 1998; Crittenden \& Claussen, 2000).

Selon la théorie de l'attachement, des modèles cognitifs opérants de soi et d'autrui formés par le jeune enfant au cours de ses premières interactions avec son parent seraient sous-jacents au déclenchement, à la répétition et à la généralisation de patrons de comportements particuliers (Bowlby, 1988; Crittenden, 1992; Crittenden \& Ainsworth, 1989; Crittenden, Lang, Claussent \& Partidge, 2000; Ramos-Marcuse \& Arsenio, 2001; Zimmermann, 2000). On pourrait donc interpréter les problèmes de comportement manifestés par les Indésirables comme des stratégies d'adaptation à une relation adverse avec leur parent ou à un environnement familial hostile et rejetant. Ce profil d'inadaptation se maintiendrait au fil du développement même si, avec la maturation et l'expérience, les processus mentaux de gestion de l'information et les stratégies comportementales associées évoluent et se complexifient (Crittenden, 2000). De fait, au fil du développement des Indésirables de cette étude, on constate une certaine évolution du comportement, mais ces stratégies plus matures ne sont pas nécessairement mieux adaptées. On peut supposer que la persistance des mauvais traitements psychologiques chez l'Indésirable entrave de façon continue le développement de stratégies d'adaptation plus adéquates. Bowlby (1988) a attribué cette difficulté à un attachement anxieux caractérisé par l'exclusion défensive des nouvelles expériences et informations incompatibles avec les modèles cognitifs préexistants.

Il reste que la qualité de l'attachement des jeunes de notre échantillon n'a pas été directement mesurée et que très peu d'études empiriques ont mis en relation à la fois la maltraitance, l'attachement et les problèmes de comportement, ce qui limite les possibilités d'interprétation de nos résultats à la lumière de la théorie de l'attachement. Aussi, l'analyse solidement documentée de Bolen (2000) montre que le soutien empirique à la théorie de l'attachement demeure équivoque et que les limites de cette théorie ne sont pas clairement définies. Dans le cas des jeunes enfants dont les besoins de base sont sévèrement négligés, menaçant leur intégrité physique et leur survie même, les résultats des études sur l'attachement pourraient être relus dans une perspective de traumatologie du développement. De Bellis (2001) mentionne n'avoir recensé aucune étude examinant le stress post traumatique chez les enfants négligés, bien que selon lui, la négligence puisse être conçue comme un trauma, tout dépendant de l'âge, du niveau de développement et de la réponse de l'enfant qui en est la cible. La perspective du trauma est exposée plus en détails suite à la description du profil-type suivant. 


\section{L'Explosif}

Ce profil s'applique particulièrement à neuf jeunes de l'échantillon, six garçons et trois filles. La famille de l'Explosif est marquée par la violence et l'abus de la figure paternelle. Il peut arriver que la mère ou la conjointe du père use aussi de violence à l'endroit des enfants, mais ses conduites abusives demeurent secondaires, voire tributaires de celles de leur conjoint. L'enfant peut être exposé à la violence conjugale, subir lui-même des abus physiques ou sexuels, ou encore vivre ces deux problématiques en juxtaposition. Habituellement, cette violence est intense, répétitive et génère un climat de peur et d'insécurité pendant toute la petite enfance et souvent aussi par la suite. À l'occasion, les tentatives de suicide ou le suicide d'un parent ajoutent au traumatisme vécu par l'enfant dans un tel contexte. La criminalité et la déviance des parents fait souvent partie de ce profil, ceux-ci affichant de lourds antécédents pouvant remonter jusqu'à leur propre enfance.

Les enfants répondent au chaos familial par une grande perturbation et une désorganisation importante sur les plans comportemental et affectif. On note une sur réactivité aux stimuli de l'environnement, une tendance aux crises, des comportements agressifs et violents, des comportements sexualisés inappropriés mais également un fond dépressif important qui s'exprime typiquement par une attitude de «je-m'en-foutisme » et un manque d'intérêts et de buts dans la vie à l'adolescence. Il semble que ce soit particulièrement chez les Explosifs que l'on observe, dès l'âge scolaire, une distinction entre filles et garçons sur le plan des problèmes de comportement, ceux des filles étant davantage intériorisés, alors que ceux des garçons apparaissent davantage extériorisés.

Alors que la négligence joue un rôle mineur dans la petite enfance de l'Explosif, elle devient de plus en plus présente lorsque le jeune vieillit. Tout se passe comme si la violence cédait graduellement le pas à une profonde désorganisation familiale, qui s'exprime principalement par un manque de supervision, un mode de vie déviant ou chaotique et un "décrochage " parental devant les comportements extrêmes de leur jeune qu'ils n'arrivent plus à encadrer. C'est sans doute pour cette raison que les Explosifs sont globalement signalés et placés plus tard que les Indésirables. Il reste que la plupart sont signalés avant l'âge de 12 ans et que tous connaissent le placement à l'adolescence. Les tableaux 4, 5 et 6 résument les résultats obtenus lors de l'analyse des dossiers.

\section{Liens avec la théorie du trauma}

La théorie du trauma pourrait contribuer à expliquer le lien entre les mauvais traitements et les problèmes de comportement chez l'Explosif. Un trauma se définit comme « le vécu direct et personnel d'un événement pouvant entraîner la mort, constituer une menace de mort ou une blessure sévère, représenter des menaces pour sa propre intégrité physique», incluant le fait d'être témoin d'une telle situation chez autrui, notamment chez un proche ou un membre de sa famille. Cette situation suscite chez l'individu «une peur intense, un sentiment d'être sans espoir ou d'horreur (chez les enfants, la réponse doit comprendre un comportement désorganisé ou 
agité) » (traduction française du DSM-IV ${ }^{8}$, American Psychiatric Association, 1996, p. 498). Or, les abus physiques et sexuels, ainsi que l'exposition à la violence conjugale, comptent parmi les traumas reconnus de l'enfance (Boney-McCoy \& Finkelhor, 1995; Milgram, 1998), de même que l'exposition aux tentatives de suicide d'un parent (Eth, 2001). Ces expériences sont typiquement vécues par les Explosifs, qui affichent d'ailleurs un comportement désorganisé.

On estime qu'entre le quart et la moitié des jeunes maltraités physiquement ou

On estime qu'entre le quart et la moitié des jeunes maltraités physiquement ou sexuellement présenteraient des symptômes de stress post traumatique.

Les chercheurs en traumatologie du développement soutiennent que, selon des mécanismes encore mal connus, l'expérience de maltraitance affecterait directement certains systèmes neurobiologiques largement interconnectés. sexuellement présenteraient des symptômes de stress post traumatique (SPT) (Bukatko \& Daehler, 2004). Ces chiffres seraient encore plus élevés chez des populations cliniques et les enfants exposés à la violence conjugale ne seraient pas épargnés (De Bellis, 2001). Les symptômes de SPT entrent dans trois grandes catégories: (1) la ré-expérimentation (souvenirs ou cauchemars répétitifs et envahissants, impression de revivre le trauma, etc.), (2) l'évitement (refus de repenser ou de parler du trauma, amnésie, perte d'intérêt, incapacité à éprouver certains sentiments, etc.) et (3) l'activation neurovégétative (irritabilité ou accès de colère, difficultés de concentration, sursauts, hypervigilance, troubles du sommeil) (American Psychiatric Association, 1996). Avec sa grande réactivité et son manque d'intérêt généralisé, l'Explosif pourrait bien correspondre au profil d'un enfant traumatisé.

Dans une enquête menée auprès d'un échantillon représentatif de jeunes Danois de 13 à 15 ans, Elklit (2002) montre que même si l'abus et la négligence comptent parmi les traumas les moins fréquemment rapportés, ce sont ceux qui sont les plus fortement associés au SPT, au même titre que le viol et les tentatives de suicide. Les traumas de type interpersonnel, notamment les agressions sexuelles et physiques, et surtout lorsque plusieurs de ces événements surviennent en juxtaposition, seraient associés à des symptômes plus sévères que les traumas de type non interpersonnel (Green, Goodman, Krupnick, Corcoran, Petty, Stockton, \& Stern, 2000). À la lumière d'une recension d'écrits, De Bellis (2001) soutient que le fait d'expérimenter un trauma sévère d'origine interpersonnelle peut outrepasser tout facteur de résilience, qu'il soit de nature génétique, constitutionnelle, sociale ou psychologique, et ainsi accroître le risque de SPT et des dysfonctions associées chez la majorité des victimes. Parmi ces problèmes associés, mentionnons les problèmes d'opposition et d'hyperactivité, surtout lorsque le trauma implique des abus physiques ou sexuels (Ford, Racusin, Ellis, Daviss, Reiser, Fleischer, \& Thomas, 2000) et les problèmes de comportement intériorisés et extériorisés (Graham-Bermann \& Levendosky, 1998; Livingston-Smith, Howard, \& Monroe, 2000), des problèmes fréquemment affichés par les Explosifs.

Pour expliquer ces liens, les chercheurs en traumatologie du développement (De Bellis, 2001; Fishbein, 2000) soutiennent que, selon des mécanismes encore mal connus, l'expérience de maltraitance affecterait directement certains systèmes neurobiologiques largement interconnectés. Les perturbations de ces systèmes se traduiraient ensuite par des problèmes de développement physique et cognitif et des

8. Le DSM-IV est la plus récente édition du Manuel diagnostique et statistique des troubles mentaux publié par I'American Psychiatric Association. Il s'agit d'une nosologie clinique qui répertorie et décrit tous les troubles mentaux en précisant leurs critères diagnostics. 
difficultés de régulation émotionnelle et comportementale, accroissant le risque de comportements inadaptés. Dans une perspective plus écologique, Harvey (1996) ajoute que l'intensité de la réponse comportementale d'un individu traumatisé est fonction d'une interaction complexe entre des facteurs individuels et des contingences environnementales. De telles hypothèses soulignent l'urgence d'investir massivement dans la prévention de la maltraitance et dans l'intervention précoce. En effet, elles induisent que le traitement des adolescents traumatisés pourrait nécessiter une synergie d'interventions pharmacologiques, psychothérapeutiques, sociales et éducatives, ce qui pose un défi énorme et extrêmement coûteux socialement.

\section{Le Délinquant}

Ce profil s'applique particulièrement à neuf jeunes de l'échantillon, trois garçons et six filles. Il s'agit du profil-type le plus difficile à documenter : les jeunes étant généralement signalés vers le début de l'adolescence, les dossiers contiennent une information limitée et parcellaire sur leurs précédentes étapes de développement. Typiquement, les parents sont plus âgés que dans les autres profils et les familles sont plus petites. Plusieurs Délinquants sont des enfants uniques et pour les autres, la fratrie est souvent composée de demi-frères ou demi-sœurs ou encore des enfants

Même lorsqu'une figure paternelle est présente, l'inefficacité chronique de l'encadrement demeure la grande constante de ce profil-type. d'un-e conjoint-e avec lesquels l'écart d'âge est important. En effet, dès la fin de la petite enfance, c'est la minorité des Délinquants qui vivent encore avec leurs deux parents et dans plusieurs cas, leur père fait peu ou pas du tout partie de leur vie. Quant à leur mère, elle présente souvent les séquelles d'une histoire de vie pénible. Envahie par ses propres difficultés, elle n'est pas à proprement parler une mère maltraitante mais plutôt une mère dépassée et démunie, ou encore fusionnelle et indifférenciée. Elle peut user occasionnellement de violence psychologique, mais c'est surtout son manque de cohérence et de fermeté dans l'application du cadre de vie qui la caractérise. Même lorsqu'une figure paternelle est présente, l'inefficacité chronique de l'encadrement demeure la grande constante de ce profil-type. Ces jeunes semblent avoir appris qu'ils peuvent tout faire sans avoir à en subir de conséquences. Ils deviennent résistants à toute forme d'encadrement et développement des problèmes d'opposition sévères et des comportements antisociaux.

Si le comportement du Délinquant n'est pas si différent de celui de l'Explosif dans ses manifestations, il est toutefois beaucoup moins désorganisé, plus structuré autour d'un mode de vie délinquant et marginal. Son agressivité semble davantage instrumentale, lui permettant d'acquérir du contrôle. Ces jeunes tendent à prendre des risques importants qui mettent leur développement et leur sécurité en danger. Ils affichent typiquement une consommation abusive de drogue qui a débuté très tôt. À l'adolescence, il n'est pas rare de voir ces jeunes impliqués dans des luttes physiques et verbales avec leurs parents. Les filles notamment paraissent vulnérables aux agressions sexuelles extra familiales et à la prostitution. L'intervention de protection se fait généralement dans une perspective d'arrêt d'agir et tous ces jeunes finissent en milieu de placement à l'adolescence. Les tableaux 7,8 et 9 résument les résultats obtenus. 
Dans la mesure où ces jeunes n'ont pas été victimes de mauvais traitements à proprement parler, on peut s'interroger sur les raisons qui ont amené les intervenant-es à les référer pour la recherche. À cet égard, il est bon de rappeler que la plupart sont des filles; socialement, il peut être plus facile d'identifier des filles au rôle de victime. De plus, parmi les trois garçons, deux présentent aussi certaines caractéristiques de l'Indésirable, surtout en ce qui concerne les manifestations de leurs problèmes de comportement. Il pourrait être plus facile d'identifier un garçon Délinquant comme une victime s'il laisse transparaître une certaine souffrance, un certain mal de vivre dans ses comportements, ce qui serait moins nécessaire chez les filles. Il pourrait donc y avoir eu un biais d'échantillonnage spécifique au profil du Délinquant.

\section{Liens avec les théories de la socialisation}

La théorie de l'apprentissage social, selon laquelle l'individu apprend par modelage et renforcement extrinsèque (Bandura, 1973; Berkowitz, 1993; Coie \& Dodge, 1998; Huesmann, 1988; Patterson, 1986), apparaît pertinente pour expliquer les problèmes de comportement du Délinquant. Dans ce modèle, la qualité du lien affectif entre le parent et l'enfant, et plus particulièrement les pratiques éducatives et disciplinaires des parents, constituent des éléments déterminants pouvant contribuer à l'émergence de problèmes de comportements chez l'enfant. Les travaux de Patterson et ses collègues $(1986,1989,1992,1995,2002)$ ont clairement démontré que les familles des jeunes qui développent, maintiennent et généralisent des comportements antisociaux se caractérisent par des attitudes extrêmement tolérantes ou permissives à l'égard des comportements inacceptables du jeune ou, à l'opposé, par des attitudes excessivement contrôlantes ou coercitives à son endroit. Dans notre étude, les familles des Délinquants sont justement caractérisées par une inefficacité chronique ou une incohérence dans l'encadrement.

Au sein d'un tel milieu familial, l'enfant développe et utilise des conduites aversives et coercitives et il apprend à exercer un contrôle sur les autres. L'absence de conséquences et l'obtention de gains par les enfants agressifs (repli du parent, évitement d'une tâche, etc.) les amèneraient à surestimer les avantages que leur procure ce type de conduite et les inciteraient à maintenir cette manière d'agir dans le futur, à la maison et à la garderie, puis à l'école avec les pairs et les adultes (Brown, Atkins, Osborne, \& Milnamow, 1996; Dodge, Lochman, Harnish, Bates, \& Pettit, 1997). Les gestes agressifs de l'enfant se manifestent à travers des visées dominatrices et de contrôle (agressivité proactive) ou encore, à travers une réaction agressive excessive face à des contrariétés (agressivité réactive), soit deux réactions typiques de nos Délinquants. Leurs difficultés de comportement se maintiennent et s'aggravent de plus en plus, une détérioration qui serait attribuable à l'exposition à des modèles violents et au renforcement de ce type de conduites à travers les interactions parents-enfants (Dodge, Bates \& Pettit, 1990; Eron, 2000; Patterson, DeBaryshe \& Ramsey, 1989). Dans ces familles, l'érosion des habiletés parentales peut être mise en lien avec des circonstances de vie difficiles telles que le chômage, la séparation, les conflits conjugaux, l'alcoolisme et la dépression (Farrington \& Loeber, 1998; Hill, 2002). 
Bref, les familles des jeunes Délinquants présentent de nombreuses caractéristiques associées aux familles dysfonctionnelles décrites plus haut. Il est clair que les jeunes de ce profil ne bénéficient pas d'un encadrement approprié et cohérent au sein de leur famille. Les modèles parentaux contrôlants ou violents semblent moins présents; par contre, l'absence fréquente de figure paternelle dès la petite enfance est à souligner. En effet, les Délinquants vivent typiquement la séparation de leurs parents en bas âge et, souvent, le contact avec leur père n'est pas maintenu. Ce sont principalement les mères qui conservent la garde exclusive de leur enfant, alors que plusieurs d'entre elles sont fragiles et souvent aux prises avec de graves difficultés personnelles (dépression, idéations suicidaires, problèmes de santé mentale), ce qui pourrait expliquer les difficultés qu'elles éprouvent à encadrer efficacement leur enfant. Devant cet échec qui se reflète dans le comportement de plus en plus incontrôlable de leur jeune, les mères peuvent en venir à adopter des attitudes psychologiquement maltraitantes à son endroit. Ce genre de dynamique relationnelle s'apparente à la dynamique "parent impuissant et enfant terrible» identifiée par Malo, Moreau, Chamberland et Roy (2003) dans une étude antérieure. De telles considérations soulignent l'urgence de dépister ces familles vulnérables et de fournir très tôt un soutien à l'exercice du rôle parental, surtout en ce qui a trait à l'application d'un cadre disciplinaire ferme, cohérent et non coercitif dès la petite enfance.

\section{Recommandations pour l'intervention en milieu scolaire}

Les résultats de la présente étude suggèrent que des jeunes affichant des comportements inadaptés et violents peuvent présenter des histoires et des dynamiques de fonctionnement distinctes, pouvant être expliquées par des cadres théoriques différents. Ce constat permet de dégager les besoins spécifiques de ces jeunes, notamment en milieu scolaire.

De par son vécu de négligence et de rejet, l'Indésirable est fragile sur le plan relationnel. Il est impératif d'éviter de le rejeter à nouveau et de le protéger du rejet de ses pairs, de l'intimidation et du taxage (Sullivan, 2000) Certaines études suggèrent que l'établissement d'un lien d'attachement significatif avec un professeur est non seulement possible (Ramos-Marcuse \& Arsenio, 2001; Howes et Ritchie, 1999), mais aussi bénéfique pour ces enfants. Cela pourrait améliorer leurs habiletés d'apprentissage, favoriser l'apparition de comportements appropriés et neutraliser l'impact des difficultés vécues à la maison (Atwool, 1999). L'établissement d'une telle relation avec un adulte signifiant pourrait bien être un élément central pour soutenir la résilience des jeunes sévèrement négligés ou abandonnés et constituer un point tournant dans leur vie (Drapeau, Saint-Jacques, Lépine, Bégin \& Bernard, 2003). Le simple fait d'accorder de l'attention à ses attitudes et ses comportements positifs et de les valider lui reflète sa valeur. Par contre, les compliments, les encouragements et les contacts physiques chaleureux peuvent susciter la méfiance, la provocation ou même l'agressivité chez l'Indésirable (Carter, 1993). Il peut être préférable de procéder de manière indirecte en invitant le jeune à s'auto-évaluer, quitte à recadrer 
son évaluation au besoin (Cartledge \& Milburn, 1995). Certes, la modification des cognitions distorsionnées qu'un individu a intégrées par rapport à lui-même, aux autres et aux relations interpersonnelles s'inscrit dans le long terme (Rubin \& Krasnor, 1986). Éventuellement, des modèles cognitifs plus justes et une meilleure estime de soi pourront servir de base au développement d'habiletés prosociales et à une meilleure insertion sociale (Cartledge \& Milburn, 1995)

Avec l'Explosif, il importe d'éviter de le remettre en contact avec le trauma de la violence, incluant les interventions coercitives et tout ce qui pourrait le prendre par surprise (contacts physiques spontanés, conséquences non prévues à l'avance, etc.). Cela risque de le faire réagir fortement, voire se désorganiser complètement (Patterson, Reid \& Dishion, 1992). Il convient de respecter les efforts d'évitement de l'Explosif et même de l'aider à se changer les idées, par exemple en l'amenant à s'impliquer dans des activités où il pourra jouer des rôles utiles et constructifs, où on lui fera confiance et où il pourra se sentir compétent et utile. Le processus de résilience des jeunes abusés pourrait bien prendre appui dans de telles activités (Drapeau et al., 2003). L'encadrement devra être ferme tout en étant chaleureux et permettre l'anticipation des événements (Kazdin, Bass, Siegel \& Thomas, 1989). L'exposition à des adultes adéquats fournit à l'Explosif des modèles d'identification et lui enseigne que la violence n'est pas nécessaire pour maîtriser sa vie. C'est sur la base d'un tel soutien qu'il pourra graduellement se réapproprier son expérience traumatisante et les émotions pénibles qui lui sont associées, l'intégrer à sa vie et lui donner un sens, accomplissant son processus de guérison (Harvey, 1996; Kennedy \& McCarthy, 1998).

Il demeure que tant l'Indésirable que l'Explosif peuvent user de violence verbale ou physique avec leurs pairs et avec les figures d'autorité. Éviter les interventions coercitives ne signifie pas qu'il faille accepter de tels comportements. Idéalement, les conséquences seront prévisibles, en proportion avec le geste posé et en lien logique avec lui (exiger qu'il répare ses torts, qu'il se retire temporairement d'une activité, etc.), et appliquées dans le calme et la modération (Kazdin et al, 1989). On devrait également prévoir une procédure à suivre afin que les crises ou les désorganisations importantes soient gérées à l'intérieur du milieu scolaire. Expulser l'enfant et le retourner dans son milieu familial est une stratégie à éviter, d'abord parce que cela revient à la rejeter, et ensuite parce que les parents de ces jeunes sont généralement négligents, rejetants ou eux-mêmes violents, ce qui ne garantit nullement une bonne prise en charge du comportement du jeune. Éventuellement, de tels jeunes pourraient être ciblés pour participer à un programme de développement des habiletés prosociales (Cartledge \& Milburn, 1995): on aborde ainsi le problème par le positif et de façon proactive, au lieu de l'aborder par la négative et de façon réactive, ce qui constitue en soi un bon exemple à donner au jeune.

Dans sa tendance à transgresser systématiquement les règles, le Délinquant peut faire usage de manipulation ou de ruse pour éviter d'assumer les conséquences de ses gestes, même lorsque les attentes à son endroit ont été préalablement clarifiées. Il importe de refuser de négocier avec lui en lui rappelant qu'il connaît la règle et d'appliquer les sanctions tel que prévu (Kazdin et al, 1989). Devant une telle contrariété, il pourra réagir par l'hostilité, l'intimidation, parfois même par la menace ou 
l'agression. De tels gestes ne devraient pas être tolérés, sous peine de renforcer un comportement inacceptable (Sullivan, 2000) Les plus graves, comme les menaces ou les voies de fait (considérées au Canada comme des infractions criminelles dans le cadre de la Loi sur les jeunes contrevenants), pourraient même faire l'objet d'un signalement ou d'une plainte aux services qui ont le pouvoir d'arrêter l'agir du jeune. Une telle intervention doit se faire dans un esprit de protection des autres élèves, du personnel de l'école, mais aussi du jeune agresseur lui-même. En effet, l'arrêt d'agir est primordial pour le Délinquant. En favorisant sa prise de conscience de l'impasse dans laquelle il se trouve, ce type d'intervention pourrait bien constituer le moteur du changement chez le jeune, un moteur alimenté par la colère, l'écoeurement ou la peur de conséquences plus importantes (Drapeau et al., 2003). De plus, l'arrêt d'agir permet au jeune d'obtenir l'aide qu'il aurait sans doute refusée si elle n'avait pas été imposée. On doit être en mesure d'intervenir auprès de ce jeune dans une perspective d'empowerment (lui seul peut choisir de changer) et de réduction des méfaits pour lui-même et pour son entourage (Allen \& Nairne, 1992). Parce que ce jeune est typiquement affilié avec des pairs antisociaux qui adoptent un mode de vie marginal, il faut l'aider à identifier des palliatifs à son ancien mode de vie (y compris la consommation de drogue), à s'y investir, à former d'autres liens sociaux et à développer ses habiletés d'affirmation devant ses anciens amis (Leblanc, 2002).

\section{Conclusion}

Cette recherche qualitative a permis de mettre en lumière trois profils-types de développement des problèmes de comportement sérieux chez des jeunes qui présentent une histoire plus ou moins lourde de mauvais traitement. À partir d'une intégration des résultats avec des théories reconnues du développement des enfants et des jeunes, il est possible de proposer des pistes concrètes d'intervention. Cette étude constitue un bel exemple d'intégration «recherche-théorie-pratique» et cela mérite d'être souligné.

Toute typologie est par essence réductrice, afin de nous aider à mieux appréhender un phénomène complexe. Dans la réalité, il est possible qu'un jeune présente à la fois des caractéristiques de l'Indésirable et de l'Explosif, ou encore de l'Indésirable et du Délinquant, sans que cela n'invalide la typologie proposée. Par contre, le fait d'identifier un jeune affichant à la fois une histoire de maltraitance et des problèmes de comportement, mais ne cadrant dans aucun des trois profils-types identifiés ici, confronterait la typologie proposée. C'est pourquoi ce genre de résultats devrait idéalement faire l'objet d'un processus de validation continu.

La typologie proposée résulte d'une co-construction qui implique tant les intervenants qui ont étoffé les dossiers de protection, les assistantes de recherche qui en ont extrait l'information requise et les chercheures qui l'ont analysée. Il est toujours possible de croire que d'autres dossiers auraient pu mener à l'identification d'autres profils-types, ou que d'autres chercheurs auraient pu voir autre chose dans le corpus de données. Toutefois, le soin consacré à l'échantillonnage et à l'atteinte de la satu- 
ration empirique, de même que la procédure de consensus appliquée lors du dépouillement des dossiers, soutiennent la valeur de la typologie proposée. De plus, cette typologie est appuyée par les résultats d'une autre étude qualitative menée en parallèle par entretiens avec des adolescent-es placé-es en ressource d'accueil (Drapeau et al., 2003). Cette étude identifie trois groupes de jeunes résilients selon le type de «point tournant» sur lequel s'appuie leur processus de résilience, soit la relation, l'action et la réflexion. Ces trois regroupements pourraient bien représenter respectivement l'Indésirable, l'Explosif et le Délinquant. On peut concevoir que ces résultats se valident mutuellement, bien que d'autres études seront nécessaires pour vérifier cette hypothèse.

En raison de la taille restreinte de l'échantillon, ce type de recherche ne permet pas la généralisation des résultats. Par contre, les résultats peuvent être transférables à toute personne qui désire les utiliser, en autant que cette personne soit capable de les situer dans son propre contexte de recherche ou d'intervention. Sans prétendre avoir développé un modèle théorique pour expliquer les problèmes de comportement sérieux des jeunes maltraités, cette étude fournit à tout le moins un cadre de référence qui contribue à notre compréhension du phénomène et qui génère des hypothèses de recherche utiles et pleines de sens pour la recherche ultérieure.

\section{Références bibliographiques}

Allem, J. \& Nairne, J. (1992). Empowering students: Classroom discussion themes for elementary school teachers and counsellors. Toronto, OISE Press Publications, University of Toronto.

American Psychiatric Association (1996). DSM-IV: manuel diagnostique et statistique des troubles mentaux (Trad : J.D. Guelfi). P.

Atwool, N. (1999). Attachment in the school setting. New Zealand Journal of Education Studies, 34, 309-322.

Bandura, A. (1973). Aggression: A social learning analysis. New York: Prentice-Hall.

Barnett, D., Manly, J.Y., \& Cichetti, D. (1993). Defining child maltreatment: The interface between policy and research. In D. Cicchetti \& S. Toth (Eds.), Child development and social policy (7-73). Norwood, NJ: Ablex Publishing.

Benda, B.B., \& Corwyn, R.F. (2002). The effect of abuse in childhood and in adolescence on violence among adolescents. Youth and Society, 33, 339-365.

Berkowitz, L. (1993). Aggression: Its causes, consequences, and control. New York: Mcgraw-Hill Book Company.

Bolen, R. (2000). Validity of attachment theory. Trauma, Violence, \& Abuse, 1, 128-153. 
Boney-McCoy, S., \& Finkelhor, D. (1995). Psychological sequelae of violent victimization in a national youth sample. Journal of Consulting and Clinical Psychology, 63, 726-736.

Bowen, F., Desbiens, N., Martin, C., \& Hamel, M. (2001). La competence sociale. In M. Hamel, L. Blanchet \& C. Martin (Eds.), 6,12,17 Nous serons bien mieux! Les déterminants de la santé et du bien-être des enfants d'âge scolaire (51-99). Québec: Les Publications du Québec.

Bowlby, J. (1988). A secure base: Parent-child attachment and healthy human development. New York: Basic books.

Brown, K., Atkins, M.S., Osborne, M.L., et Milnamow, M. (1996). A revised teacher rating scale for reactive and proactive aggression. Journal of Abnormal Child Psychology. 24, 473-480.

Bukatko, D., \& Daehler, M.W. (2004). Child development. A thematic approach, $5^{\text {th }}$ edition. New York: Houghton Mifflin Company.

Camic, P.M., Rhodes, J.E., \& Yardley, L. (2003). Naming the stars: Integrating qualitative methods into psychological research. In P.M. Camic, J.E. Rhodes \& L. Yardley (Eds.), Qualitative research in psychology. Expanding perspectives in methodology and desing (3-15). Washington, DC: American Psychological Association.

Carlson, B.E. (1991). Outcome of physical abuse and observation of marital violence among adolescents in placement. Journal of Interpersonal Violence, 6, 526-534.

Carter, J.F. (1993). Self-management. Teaching exceptional children, 25, 28-32.

Cartledge, G. \& Milburn, J.F. (1995). Teaching social skills to children and youth: Innovative approaches. Massachusetts: Allyn \& Bacon.

Cicchetti, D., Rogosch, F.A. \& Toth, S.L. (1998). Maternal depressive disorder and contextual risk: Contributions to the development of attachment insecurity and behavior problems in toddlerhood. Development and Psychopathology, $10,283-300$.

Cicchetti, D.,\& Toth, S.L. (1995). A developmental psychopathology perspective on child abuse and neglect. Journal of the American Academy of Child and Adolescent Psychiatry, 42, 541-565.

Claussen, A.H., \& Crittenden, P.M. (1991). Physical and psychological maltreatment: Relations among types of maltreatment. Child Abuse \& Neglect, 15, 5-18.

Cole-Detke, H., \& Kobak, R. (1998). The effects of multiple abuse in interpersonal relationships : An attachement perspective. Journal of Aggression, Maltreatment and Trauma, 2, 189-205.

Craig, W.M., Peters, R.D., \& Kornaski, R. (1998). L'intimidation et la victimisation chez les enfants d'âge scolaire au Canada. Développement des ressources humaines Canada: Direction générale de la recherche appliquée, Politique stratégique. 
Coie, J.D., \& Dodge, K.A. (1998). Aggression and antisocial behavior. In W. Damon \& N. Eisenberg (Eds.), Handbook of Child Psychology . vol.3 - Social, Emotional and Personality Development (779-862). New York: Wiley.

Cooper, M.L., Shaver, P.R., \& Collins, N.L. (1998). Attachment styles, emotion regulation, and adjustment in adolescence. Journal of Personality and Social Psychology, 74, 1380-1397.

Crittenden, P.M. (2000). A dynamic-maturational approach to continuity and change in pattern of attachment. In P.M. Crittenden \& A.H. Claussen (Eds.), The organization of attachment relationships: Maturation, culture, and context. New York: Cambridge University Press, 343-357.

Crittenden, P.M. (1992). Children's strategies for coping with adverse home environments : an interpretation using attachment theory. Child Abuse \& Neglect, 16, 329-343.

Crittenden, P.M., \& Ainsworth, M.D.S. (1989). Child maltreatment and attachment theory. In D. Cicchetti \& V. Carlson (Eds), Child maltreatment: Theory and research on the causes and consequences of child abuse and neglect. New York: Cambridge University Press, 432-463.

Crittenden, P.M., \& Claussen, A.H. (2000). Adaptation to varied environments. In P.M. Crittenden \& A.H. Claussen (Eds.), The organization of attachment relationships: Maturation, culture, and context. New York: Cambridge University Press, 234-262.

Crittenden, P.M., Claussen, A.H., \& Sugarman, D.B. (1994). Physical and psychological maltreatment in middle childhood and adolescence. Development and Psychopathology, 6, 145-164.

Crittenden, P.M., Lang, C., Claussen, A.H., \& Partridge, M.F. (2000). Relations among mothers' dispositional representations of parenting. In P.M. Crittenden \& A.H. Claussen (Eds.), The organization of attachment relationships : Maturation, culture, and context. New York: Cambridge University Press, 214-233.

D’Amours, Y. (1995). Le point sur la délinquance et le suicide chez les jeunes. Québec: Conseil permanent de la jeunesse.

Debarbieux, É. (mai 2003). La violence à l'école : une mondialisation? Deuxième conférence mondiale sur la violence à l'école, Québec, Canada.

De Bellis, M.D. (2001). Developmental traumatology: The psychobiological development of maltreated children and its implications for research, treatment, and policy. Development and Psychopathology, 13, 539-564.

Deslandes, R. (2001). L'environnement scolaire. In M. Hamel, L. Blanchet \& C. Martin (Eds.), 6,12,17 Nous serons bien mieux! Les déterminants de la santé et du bien-être des enfants d'âge scolaire (251-286). Québec: Les Publications du Québec. 
Dishion, T. J., French, D. C., \& Patterson, G. R. (1995). The development and ecology of antisocial behavior. In D. Cicchetti \& D. J. Cohen (Eds.), Development and Psychopathology, Vol.2. New-York: Wiley.

Dobkin, P.L., Tremblay, R.E., Masse, L.-C., \& Vitaro, F. (1995). Individual and peer characteristics in predicting boys' early onset of substance abuse: A seven-year longitudinal study. Child Development, 66, 1198-1214.

Dodge, K.A., Bates, J.E., \& Petit, G.S. (1990). Mechanisms in the cycle of violence. Science, 250, 1678-1682.

Dodge, K.A, Lochman, J.E., Harnish, J.D., Bates, J.E., \& Pettit, G.S. (1997). Reactive and proactive aggression in school children and psychiatrically impaired chronically assaultive youths. Journal of Abnormal Psychology, 106, 37-51.

Drapeau, S., Saint-Jacques, M.-C., Lépine, R., Bégin, G., \& Bernard, M. (2003). La résilience chez les adolescents placés : l'action, les relations, la réflexion. Rapport de recherche. Université Laval, Québec: Centre de recherche sur l'adaptation des jeunes et des familles à risque.

Dryfoos, J. G. (1990). Adolescents at risk: Prevalence and prevention. New-York: Oxford University Press.

Elklit, A. (2002). Victimization and PTSD in a Danish national youth probability sample. Journal of American Academy of Child and Adolescent Psychiatry, 41, 174-181.

Erickson, M., \& Egeland, B. (1987). A developmental view of the psychological consequences of maltreatment. School Psychology Review, 16, 156-168.

Eron, L.D. (2000). A psychological perspective. In Van Hasselt, V. B., \& Hersen, M. (Eds), Aggression and violence: An introductory text (23-39). Needham Heights, MA: Allyn \& Bacon.

Eth, S. (2001). Childhood Trauma in perspective. In S. Eth (Ed.), PTSD in children and adolescents (introduction). Washington, DC: American Psychiatric Publishing.

Farrington, D.P., \& Loeber, R. (2000). Epidemiology of juvenile violence. Child and Adolescent Psychiatric Clinics of North America, 9, 733-748.

Finkelhor, D., \& Dziuba-Leatherman, J. (1994). Victimization of children. American Psychologist, 49(3), 173-183.

Finzi, R., Cohen, O., Sapir, Y., \& Weizman, A. (2000). Attachment styles in maltreated children : A comparative study. Child Psychiatry and Human Development, 31, 113-128.

Fishbein, D.H. (2000). How can neurobiological research inform prevention strategies? In D.H. Fishbein (Ed.), The science, treatment, and prevention of antisocial behaviours. Application to the criminal justice system (chap. 25). Kingston, NJ: Civic Research Institute. 
Ford, J.D., Racusin, R., Ellis, C.G., Daviss, W.B., Reiser, J., Fleisher, A., \& Thomas, J. (2000). Child maltreatment, other trauma exposure, and posttraumatic symptomatology among children with oppositional defiant and attention deficit hyperactivity disorder. Child Maltreatment, 5, 205-217.

Forget, G., Bilodeau, A., \& Tétreault, J. (1992). Facteurs reliés à la sexualité et à la contraception chez les jeunes et décrochage scolaire: Un lien insolite mais réel. Apprentissage et Socialisation, 15, 29-38.

Gagné, M.-H, \& Bouchard, C. (sous presse). Family dynamics associated with the use of psychologically violent parental practices. Journal of Family Violence, 19 (2).

Garbarino, J. (1987). What can the school do on behalf of the psychologically maltreated child and the community? School Psychology Review, 16, 181-187.

Graham-Bermann, S.A., \& Levendosky, A.A. (1998). Traumatic stress symptoms in children of battered women. Journal of Interpersonal Violence, 13, 111-128.

Green, B.L., Goodman, L.A., Krupnick, J.L., Corcoran, C.B., Petty, R.M., Stockton, P., \& Stern, N.M. (2000). Outcomes of single versus multiple trauma exposure in a screening sample. Journal of Traumatic Stress, 13, 271-286.

Harris, M., \& Fallot, R.D. (2001). Envisioning a trauma-informed service system: a vital paradigm shift. In M. Harris \& R.D. Fallot (Eds.), Using trauma theory to design service systems. San Francisco, CA: Jossey-Bass / New Directions for Mental Health Services, no 89.

Hart, S.N. (1988). Psychological maltreatment: emphasis on prevention. School Psychology International, 9, 243-255.

Harvey, M.R. (1996). An ecological view of psychological trauma and trauma recovery. Journal of Traumatic Stress, 9, 3-23.

Hawkins, J.D., Catalano, R.F., \& Miller, J.Y. (1992). Risk and protective factors for alcohol and other drug problems in adolescence and early adulthood: Implications for substance abuse prevention. Psychological Bulletin, 112, 64-102.

Hill, J. (2002). Biological, psychological and social processes in the conduct disorders. Journal of Child Psychology and Psychiatry, 43, 133-164.

Higgins, D.J., \& McCabe, M.P. (2000). Multi-type maltreatment and the long-term adjustment of adults. Child Abuse Review, 9, 6-18.

Howes, C., \& Ritchie, S. (1999). Attachment organizations in children with difficult life circumstances. Development and Psychopathology, 11, 251-268.

Huesmann, L.R. (1988). An information processing model for the development of agression. Aggressive Behavior, 14, 13-24.

Jonson-Reid, M. (1998). Youth violence and exposure to violence in childhood: An ecological review. Aggression and Violent Behavior, 3, 159-179. 
Kauffman, J.M. (1997). Conclusion: A little of everything, a lot of nothing is an agenda for failure. Journal of Emotional and Behavioral Disorders, 5, 76-81.

Kennedy, M.R. (1991). Homeless and runaway youth mental health issues : No access to the system. Journal of Adolescent Health, 12, 576-579.

Kennedy, J., \& McCarthy, C.J. (1998). Bridging Worlds. Understanding and facilitating adolescent recovery form the trauma of abuse. New York: The Haworth Maltreatment and Trauma Press.

King, A.J.C., Boyce, W.F., \& King, M.A. (1999). La santé des jeunes: tendances au Canada. Ottawa: Santé Canada.

Kolbo, J.R., Blakely, E.H., \& Engleman, D. (1996). Children who witness domestic violence: A review of empirical litterature. Journal of Interpersonal Violence, 11, 281-293.

Leblanc, M. (2002). Intervenir autrement...un modèle différentiel pour les adolescents en difficulté. Boucherville, Québec: G. Morin.

Livingston-Smith, S.L., Howard, J.A., \& Monroe, A.D. (2000). Issues underlying behaviour problems in at-risk adopted children. Children and Youth Services Review, 22, 539-562.

McGee, R.A., Wolfe, D.A., \& Wilson, S.K. (1997). Multiple maltreatment experiences and adolescent behavior problems: Adolescents' perspectives. Development and Psychopathology, 9, 131-149.

Malo, C., Moreau, J., Chamberland, C., Roy, C. (2003) Psychological maltreatment of preschoolers: Four profiles of parent-child interaction associated with various cognitive, emotional, and behavioral characteristics. In K. Kufeldt \& B. McKenzie (Eds.), Child Welfare: Connecting research, policy, and practice. Waterloo, Ont.: Wilfrid Laurier University Press.

Miles, M.B., \& Huberman, A.M. (1994, 2003). Analyse des données qualitatives. (traduction de la $2^{\mathrm{e}}$ édition américaine). Paris : De Boeck.

Milgram, N.A. (1998). Children under stress. In T.H. Ollendick \& M. Hersen (Eds.), Handbook of child psychopathology, $3^{\text {rd }}$ edition (505-533). New York: Plenum Press.

Morton, N., \& Browne, K.D. (1998). Theory and observation of attachment and its relation to child maltreatment: A review. Child Abuse \& Neglect, 22, 1093-1104.

Moss, E., Rousseau, D., Parent, S., St-Laurent, D., \& Saintonge, J. (1998). Correlates of attachement at school age : Maternal reported stress, mother-child interaction, and behavior problems. Child Development, 69, 1390-1405.

Moss, E., St-Laurent, D., Rousseau, D., Parent, S., Gosselin, C., \& Saintonge, J. (1999). L'attachement à l'âge scolaire et le développement des troubles de comportement. Revue canadienne des sciences du comportement, 31, 107-118. 
Mucchielli, A. (1996). Dictionnaire des méthodes qualitatives en sciences humaines et sociales. Armand Colin.

O'Keefe, M. (1995). Predictors of child abuse in maritally violent families. Journal of Interpersonal Violence, 10, 3-25.

Patterson, G.R. (1986). Performance models for antisocial boys. American Psychologist, 41, 728-731.

Patterson, G.R., DeBaryshe, B.D., \& Ramsey, E. (1989). A developmental perspective on antisocial behavior. American Psychologist, 44, 329-335.

Patterson G. R., Reid, J. B., \& Dishion, T. J. (1992). A social learning approach: Vol.4. Antisocial boys. Eugene, OR: Castilla.

Pierrehumbert, B., Miljkovitch, R., Plancherel, B., Halfon, O., \& Ansermet, F. (2000). Attachment and temperament in early childhood; Implications for later behavior problems. Infant and Child Development, 9, 17-32.

Pirès, A.P. (1997). Échantillonnage de recherche qualitative: essai théorique et méthodologique. In J. Poupart, J.P. Deslauriers, L.H. Groulx, A. Laperrière, R. Mayer \& A.P. Pirès (Eds.), La recherche qualitative. Enjeux épistémologiques et méthodologiques. Boucherville: Gaëtan Morin.

Ramos-Marcuse, F., \& Arsenio, W.F. (2001). Young children's emotionally charged moral narratives : Relations with attachment and behavior problems. Early Education and Development, 12, 165-184.

Reid, J.B., Patterson, G.R., \& Snyder, J. (Eds.) (2002). Antisocial behavior in children and adolescents. A developmental analysis and model for intervention. Washington, DC: American Psychological Association.

Reppucci, N.D., Fried, C.S., \& Schmidt, M.G. (2002). Youth violence: Risk and protective factors. In R.R. Corrado, R. Roesch, S.D. Hart, \& J.K. Gierowski (Eds.), Multi-problem violent youth: A foundation for comparative research on needs, interventions and outcomes (3-22). Amsterdam: IOS Press.

Rubin, K.H. \& Krasnor, L.R. (1986). Social-cognitive and social behavioural perspectives on problem solving. Dans M. Perlmutter (Ed.) Cognitive perspectives on children's social and behavioural development. Hillsdale, NJ: Lawrence Erlbaum Associates.

Rumberger, R.W. (1995). Dropping out of middle school: A multilevel analysis of students and schools. American Educational Research Journal, 32, 583-625.

Sullivan, K. (2000). The anti-bullying handbook. Oxford: University Press.

Tourigny, M., Mayer, M., Wright, J., Lavergne, C., Trocmé, N., Hélie, S., Bouchard, C., Chamberland, C., Cloutier, R., Jacob, M., Boucher, J., \& Larrivée, M.-C. (2002). Étude sur l'incidence et les caractéristiques des situations d'abus, de négligence, d'abandon et de troubles de comportement sérieux signalées à la Direction de la protection de la jeunesse au Québec (ÉIQ). Montréal : Centre de liaison sur l'intervention et la prévention psychosociales (CLIPP). 
Tousignant, M. (1993). Famille, écologie sociale et comportements suicidaires à l'école secondaire. Montréal : Laboratoire de recherche en écologie humaine et sociale. Rapport de recherche au CQRS.

Trickett, P.K., \& Putnam, F.W. (1998). Developmental consequences of child sexual abuse. In P.K. Trickett \& C.J. Schellenbach (Eds.), Violence against children in the family and the community. Washington, DC: American Psychological Association.

Vissing, Y.M., Straus, M.A., Gelles, R.J., \& Harrop, J.W. (1991). Verbal aggression by parents and psychosocial problems of children. Child Abuse \& Neglect, 15, 223-238.

Wenar, C., \& Kerig, P. (2000). Developmental psychopathology: From infancy through adolescence, fourth edition. New York : McGraw-Hill.

Zimmermann, P. (2000). L’attachement à l'adolescence: mesure, développement et adaptation. In G.M. Tarabulsy, S. Larose, D.R. Pederson \& G. Moran (Eds.), Attachement et développement : le rôle des premières relations dans le développement humain. Enfance, 7 : 181-204.

\section{Note des auteures}

Cette recherche a été réalisée grâce à une subvention du Fonds Richelieu de recherche sur l'enfance, concours 2000-2002.

Les auteures désirent remercier, à l'Université Laval, Égide Royer pour sa contribution à l'obtention de financement pour cette recherche, de même que Anne-Marie Barrette, Simon Lapierre, Mélissa Lasnier et Dominique Rioux, assistants de recherche; au Centre jeunesse de Québec - Institut universitaire, Carole Laquerre, Guylaine Duchêne, Richard Carrier, Marie-France Émond et Serge Grenier, pour leur soutien à la réalisation du projet, Lucie Bédard et Jacques Fournier pour la validation de la grille d'analyse des dossiers, et Valérie Houle pour son travail de transfert de connaissances dans le milieu. 


\section{Tableau 1. Famille et intervention de protection chez l'Indésirable}

\begin{tabular}{|c|c|c|}
\hline Antécédents et $0-5$ ans $(n=5)$ & $6-11$ ans $(n=5)$ & $12-17$ ans $(n=4)^{a}$ \\
\hline $\begin{array}{l}\text { Chez les filles, peu d'antécédents } \\
\text { parentaux sont notés. } \\
\text { Chez les garçons, on note des mères } \\
\text { lourdement taxées : } \\
\text { - Enfance difficile ( } 3 / 3) \\
\text { - Placées dans leur jeunesse }(2 / 3) \\
\text { - Ont eu un enfant avant } 18 \text { ans } \\
\text { (2/3); une autre s'est mariée à } \\
17 \text { ans et a subi de la violence } \\
\text { conjugale } \\
\text { - Sont dépressives et suicidaires } \\
\text { (2/3): } \\
\text { * Ces deux mères demeureront dans } \\
\text { cet état jusqu'à l'adolescence de leur } \\
\text { enfant. } \\
\text { En général, mères ont eu d'autres } \\
\text { enfants avant (5/5) et après (3/5): } \\
\text { jeunes parents, familles nombreuses. } \\
\text { Discontinuités relationnelles: } \\
3 / 5 \text { sont déjà signalés ou placés, et } \\
1 \text { garçon ne verra plus son père } \\
\text { suite au divorce de ses parents. }\end{array}$ & $\begin{array}{l}\text { - Nombreux placements et tentatives } \\
\text { de réinsertion échouées }(5 / 5) \\
\text { - Parents non collaborateurs, refusent } \\
\text { de se remettre en question ou nient } \\
\text { leurs responsabilités ou problèmes } \\
(3 / 5) \\
\text { Les filles vivent plus de rejet explicite } \\
\text { de la part de leurs parents (voir } \\
\text { tableau } 2 \text { ). } \\
\text { Les garçons vivent plus d'instabilité } \\
\text { relationnelle avec leurs parents: } \\
\text { - Mère dépressive et suicidaire }(2 / 3) \\
\text { - Contacts irréguliers ou inexistants } \\
\text { avec un ou deux parents ( } 3 / 3 \text { ) } \\
\text { Les problèmes observés ici se poursui- } \\
\text { vront généralement à l'adolescence... }\end{array}$ & $\begin{array}{l}\text { - Placements - la suite de ce qu'ils ont } \\
\text { vécu avant (4/4) } \\
\text { Parmi les } 2 \text { garçons qui ont une mère } \\
\text { dépressive et suicidaire, I'une finit par } \\
\text { se suicider alors qu'il a } 12 \text { ans. } \\
\text { À terme, on observe seulement une } \\
\text { famille biparentale intacte sur } 5 \text {. }\end{array}$ \\
\hline
\end{tabular}

a. une participante n'a pas atteint l'âge de 12 ans au moment du recueil des données 
Tableau 2. Mauvais traitements chez l'Indésirable

\begin{tabular}{|c|c|c|}
\hline $0-5$ ans $(n=5)$ & $6-11$ ans $(n=5)$ & $12-17$ ans $(n=4)^{a}$ \\
\hline $\begin{array}{l}\text { Mauvais traitements psychologiques } \\
\text { graves }(5 / 5) \text { : } \\
\text { - Rejet, hostilité } \\
\text { - Abandon pour périodes indéter- } \\
\text { minées } \\
\text { - Exposition à des tentatives de } \\
\text { suicide } \\
\text { Négligence grave }(3 / 5) \text { : } \\
\text { - Abandon sans voir à ce que les } \\
\text { besoins de l'enfant soient comblés } \\
\text { - Absence totale de supervision } \\
\text { - Parents ne font pas ce qu'il faut pour } \\
\text { régler les problèmes de l'enfant } \\
\text { - Signes de malnutrition chez l'enfant } \\
\text { - Symptômes de sevrage à la } \\
\text { naissance chez l'enfant } \\
\text { Abus sexuel: } \\
\text { - Doute chez une fille (père) et un } \\
\text { garçon (mère) } \\
\text { - Une fille a été exposée à du } \\
\text { matériel pornographique et à la } \\
\text { sexualité des parents }\end{array}$ & $\begin{array}{l}\text { Poursuite des mauvais traitements } \\
\text { psychologiques graves }(4 / 5) \text {, souvent } \\
\text { accompagnées d'autres manifestations } \\
\text { de sévérité variée. } \\
\text { L'autre }(1 / 5) \text { subit un renversement de } \\
\text { rôles mère / enfant. } \\
\text { Négligence s'atténue, mais peut } \\
\text { demeurer sous des formes plus } \\
\text { mineures (propreté, salubrité), ou : } \\
\text { - manque de supervision }(1 / 5) \\
\text { - Ne font pas ce qu'il faut pour régler } \\
\text { les problèmes de l'enfant ( } 1 / 5) \\
\text { - exposition à des modèles déviants } \\
\text { (2/5) } \\
\text { Abus physique mineurs ( } 3 / 5) \text {, } \\
\text { pouvant être reliés à un profil général } \\
\text { de rejet et d'hostilité. } \\
\text { Abus sexuel: } \\
\text { - On continue d'avoir des doutes au } \\
\text { sujet de la mère d'un garçon }\end{array}$ & $\begin{array}{l}\text { Mauvais traitements psychologiques } \\
\text { et négligence : } \\
\text { - Désinvestissement et désintéresse- } \\
\text { ment généralisé des parents (4/4) } \\
\text { - Chez une fille, une dynamique de } \\
\text { rejet affectif grave se poursuit } \\
\text { depuis la petite enfance } \\
\text { - Un garçon vit le suicide de sa mère } \\
\text { à } 12 \text { ans, alors que son père investit } \\
\text { minimalement avec lui, omettant de } \\
\text { faire ce qu'il faut pour régler les } \\
\text { problèmes de son fils. } \\
\text { - Un autre garçon est exposé à des } \\
\text { modèles déviants par le biais de ses } \\
\text { parents (délinquance, toxicomanie, } \\
\text { incarcérations) }\end{array}$ \\
\hline
\end{tabular}

a. une participante n'a pas atteint l'âge de 12 ans au moment du recueil des données 
Tableau 3. Problèmes de comportement chez l'Indésirable

\begin{tabular}{|c|c|c|}
\hline $0-5$ ans $(n=5)$ & $6-11$ ans $(n=5)$ & $12-17$ ans $(n=4) a$ \\
\hline $\begin{array}{l}\text { - Agressivité verbale et physique } \\
(4 / 5) \\
\text { - Grande agitation ou hyperactivité } \\
(4 / 5) \\
\text { - Énurésie ou encoprésie, peut aller } \\
\text { jusqu'à manger ses selles }(3 / 5) \\
\text { Chez les garçons, recherche d'attention } \\
(2 / 3) \\
\text { Chez les filles, possessivité, jalousie et } \\
\text { grand besoin d'exclusivité }(2 / 2) \\
\text { En général, tous ces problèmes tendent } \\
\text { à se maintenir à l'âge scolaire... }\end{array}$ & $\begin{array}{l}\text { - Opposition }(5 / 5) \\
\text { - Agressivité verbale et physique } \\
(4 / 5) \\
\text { - Agitation, hyperactivité, manque } \\
\text { d'attention ou de concentration } \\
(4 / 5) \\
\text { - Possessivité, difficulté à partager, } \\
\text { besoin d'exclusivité }(3 / 5) \\
\text { - Énurésie ou encoprésie }(3 / 5) \\
\text { Il s'agit là des manifestations les plus } \\
\text { communes, mais elles sont très variés } \\
\text { à cet âge, incluant tant des problèmes } \\
\text { intériorisés qu'extériorisés. }\end{array}$ & $\begin{array}{l}\text { - Opposition ( } 3 / 4) \\
\text { - Tendance à minimiser et banaliser } \\
\text { ses problèmes, non collaboration } \\
(4 / 4) \\
\text { - Difficulté à exprimer ses émotions, } \\
\text { renfermé }(3 / 4) \\
\text { - Idéations ou tentatives de suicide* } \\
(3 / 4) \\
\text { On note également que } 2 / 3 \text { garçons } \\
\text { affichent les problèmes } \\
\text { suivants : } \\
\text { - Consommation ou trafic de } \\
\text { drogues*: } \\
\text { - Arrogance et impolitesse } \\
\text { - Manipulation } \\
\text { - Sentiment d'injustice subie } \\
\text { - Délits } \\
\text { - Impulsivité } \\
\text { - Agressivité } \\
\text { - Symptômes de personnalité limite } \\
\text { - Tendance au contrôle et à } \\
\text { l'intimidation } \\
\text { * La plus jeune participante avait } \\
\text { aussi fait des menaces et une tentative } \\
\text { de suicide dès } 8 \text { ans. } \\
\text { ** chez un participant, la consom- } \\
\text { mation avait commencé dès l'âge } \\
\text { scolaire. }\end{array}$ \\
\hline
\end{tabular}

a. une participante n'a pas atteint l'âge de 12 ans au moment du recueil des données 
Tableau 4. Famille et intervention de protection chez l'Explosif

\begin{tabular}{|c|c|c|}
\hline Antécédents et $0-5$ ans $(n=9)$ & $6-11$ ans $(n=9)$ & $12-17$ ans $(n=5)^{a}$ \\
\hline $\begin{array}{l}\text { Antécédents des parents pas } \\
\text { systématiquement documentés, mais : } \\
\text { - histoires de criminalité, trafic, } \\
\text { prostitution... } \\
\text { - histoires de violence familiale, } \\
\text { d'alcoolisme, de santé mentale des } \\
\text { parents et de placements } \\
\text { La violence conjugale, provenant du } \\
\text { père ou d'un nouveau conjoint, est un } \\
\text { thème central : } \\
\text { - } 4 / 6 \text { garçons exposés } \\
\text { - } 2 / 3 \text { filles exposées, mais surtout } \\
\text { sous forme de climat de contrôle } \\
\text { extrême } \\
\text { - } 1 \text { garçon exposé à un climat } \\
\text { familial de violence et d'hostilité } \\
\text { généralisées } \\
\text { Discontinuités relationnelles: } \\
\text { - Séparation des parents (4/9) } \\
\text { - Suicide du père (1/9) } \\
\text { - } 1 \text { er signalement retenu (3/9) } \\
\text { - Placement (1/9) }\end{array}$ & 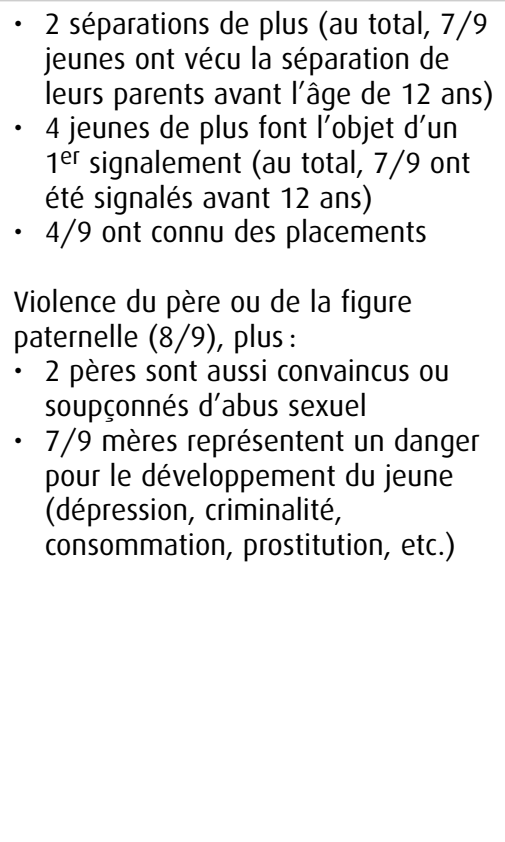 & $\begin{array}{l}\text { - Placements }(5 / 5) \\
\text { - Familles dysfonctionnelles }(5 / 5) \\
\text { Chez les jeunes qui ont atteint } \\
\text { I'adolescence }(5 / 9) \text {, on remarque un } \\
\text { maintien de la problématique de la } \\
\text { famille, ou encore une démission des } \\
\text { parents, ce qui conduit invariablement } \\
\text { au placement. }\end{array}$ \\
\hline
\end{tabular}

a. Quatre participant-es n'ont pas atteint l'âge de 12 ans au moment du recueil des données 
Tableau 5. Mauvais traitements chez l'Explosif

\begin{tabular}{|c|c|c|}
\hline $0-5$ ans $(n=9)$ & $6-11$ ans $(n=9)$ & $12-17$ ans $(n=5)^{a}$ \\
\hline $\begin{array}{l}\text { Mauvais traitements psychologiques : } \\
\text { - Exposition à la violence conjugale } \\
(6 / 9), \text { pouvant être doublés de } \\
\text { l'abandon paternel }(1 / 9) \text {, d'aliéna- } \\
\text { tion parentale (1/9) ou d'exposition } \\
\text { aux tentatives de suicide de la mère } \\
(1 / 9) \\
\text { Abus physique": } \\
\text { - } 6 / 9 \text { traités avec dureté sur le plan } \\
\text { physique, ou doute qu'ils l'ont été, } \\
\text { la plupart du temps par le père ou } \\
\text { le conjoint de la mère. } \\
\text { * Difficile d'estimer la sévérité de } \\
\text { l'abus car souvent, l'information est } \\
\text { obtenue a posteriori. } \\
\text { Abus sexuel: } \\
\text { - } 3 / 3 \text { filles et } 1 / 6 \text { garçon abusés } \\
\text { sexuellement, ou doute qu'ils l'ont } \\
\text { été, dont } 3 / 4 \text { par leur père. } \\
\text { Un seul de ces abus sexuel a été } \\
\text { prouvé, mais plus tard. } \\
\text { Négligence : pas complètement } \\
\text { absente du portrait, mais une minorité } \\
\text { en ont vécu (4/9) et sous des formes } \\
\text { mineures. }\end{array}$ & $\begin{array}{l}\text { Mauvais traitements psychologiques } \\
\text { (8/9) assez sévères et diversifiés: } \\
\text { - exposition à la violence conjugale, } \\
\text { aliénation parentale, hostilité, rejet, } \\
\text { abandon, désinvestissement. } \\
\text { - Peut venir de père, mère, nouveau } \\
\text { ou nouvelle conjoint-e. } \\
7 / 9 \text { traités durement, voire abusés } \\
\text { physiquement par père ou conjoint de } \\
\text { la mère, parfois aussi par mère ou } \\
\text { conjointe du père (2/9). } \\
\text { Non seulement continuité, mais } \\
\text { aggravation globale des abus } \\
\text { psychologiques et physiques... } \\
\text { cas simplement mieux documentés? } \\
\text { 4/9 abusés sexuellement, ou } \\
\text { doute de tels abus. } \\
\text { dans } 2 \text { cas, père (continuité avec } \\
\text { petite enfance) } \\
\text { - dans } 2 \text { autres cas, revictimisation } \\
\text { dans un contexte extra-familial. } \\
1 \text { garçon exposé à sexualité de } \\
\text { mère. } \\
\text { Négligence : mineure. Typiquement, } \\
\text { parents ne protègent pas l'enfant ou } \\
\text { ne font pas ce qu'il faut pour régler ses } \\
\text { problèmes (4/9). Malpropreté chez } \\
\text { un garçon. }\end{array}$ & $\begin{array}{l}\text { Mauvais traitements psychologiques } \\
(5 / 5) \text {. Typiquement, père peu investis } \\
\text { auprès de leur jeune, ou l'ont aban- } \\
\text { donné. Mères plus présentes, mais } \\
\text { davantage agressantes et rejetantes. } \\
\text { Abus physique presque disparu, } \\
\text { excepté chez } 1 \text { fille qui continue à être } \\
\text { frappée par son père et ses frères. } \\
\text { Aucun abus sexuel, sauf une situation } \\
\text { de corruption où } 1 \text { fille est incitée à se } \\
\text { prostituer par sa mère. } \\
\text { Négligence (5/5) a pris une ampleur } \\
\text { considérable, assez sévère, sous } \\
\text { forme de: } \\
\text { - manque de supervision } \\
\text { - absence d'encadrement } \\
\text { - omission à faire ce qu'il faut pour } \\
\text { régler les problèmes du jeune } \\
\text { - exposition à des modèles déviants } \\
\text { ou même permission ou encourage- } \\
\text { ments à participer à des activités } \\
\text { illicites }\end{array}$ \\
\hline
\end{tabular}

a. Quatre participant-es n'ont pas atteint l'âge de 12 ans au moment du recueil des données 
Tableau 6. Problèmes de comportement chez l'Explosif

\begin{tabular}{|c|c|c|}
\hline $0-5$ ans $(n=9)$ & $6-11$ ans $(n=9)$ & $12-17$ ans $(n=5)^{a}$ \\
\hline $\begin{array}{l}\text { Pour } 6 / 9 \text { jeunes, aucun problème de } \\
\text { comportement n'est documenté avant } \\
\text { l'âge de } 6 \text { ans. } \\
\text { Chez les } 3 \text { autres, les problèmes les } \\
\text { plus communs sont: } \\
\text { - Comportements sexualisés et } \\
\text { intérêt pour la sexualité }(3 / 3) \\
\text { - Problèmes d'opposition }(2 / 3) \\
\text { Lorsque de tels problèmes sont } \\
\text { identifiés, ils se maintiennent à l'âge } \\
\text { scolaire. }\end{array}$ & $\begin{array}{l}\text { - Comportements sexualisés et } \\
\text { intérêt pour la sexualité }(6 / 9) \\
\text { - Problèmes d'opposition }(6 / 9) \\
\text { On remarque également chez la } \\
\text { plupart des garçons: } \\
\text { - Agressivité, conflits et bagarres } \\
(5 / 6) \text {, souvent doublé d'un manque } \\
\text { d'autocontrôle }(3 / 6) \text { ou d'anxiété } \\
(3 / 6)\end{array}$ & $\begin{array}{l}\text { - Perturbation, désorganisation } \\
\text { émotive ou comportementale } \\
\text { extrêmes }(4 / 5) \\
\text { - Fugues et menaces de fugues (4/5) } \\
\text { - Vols et autres délits }(4 / 5) \\
\text { - Problèmes d'opposition }(3 / 5) \\
\text { - Manque d'intérêt généralisé, } \\
\text { absence de buts }(3 / 5) \\
\text { Ce que les filles ont en commun : } \\
\text { - Perte d'appétit }(2 / 2) \\
\text { - Tendance à mentir }(2 / 2) \\
\text { - Isolement social }(2 / 2) \\
\text { - Très renfermées }(2 / 2) \\
\text { Ce que les garçons ont en commun: } \\
\text { - Agressivité et violence }(3 / 3) \\
\text { - Consommation ou trafic }(2 / 3) \\
\text { - Déficit d'attention et hyperactivité } \\
\text { (2/3) } \\
\text { - Non collaboration }(2 / 3) \\
\text { - Vandalisme }(2 / 3) \\
\text { - Provocation }(2 / 3)\end{array}$ \\
\hline
\end{tabular}

a. Quatre participant-es n'ont pas atteint l'âge de 12 ans au moment du recueil des données 
Tableau 7. Famille et intervention de protection chez le Délinquant

\begin{tabular}{|c|c|c|}
\hline Antécédents et 0-5 ans $(n=9)$ & $6-11$ ans $(n=9)$ & $12-17$ ans $(n=9)$ \\
\hline $\begin{array}{l}\text { Aucune information sur les antécédents } \\
\text { des parents pour } 3 / 9 \text { dossiers. } \\
\text { Pour les autres, très peu d'information } \\
\text { disponible. } \\
\text { - Parent plutôt âgés ( } 3 / 6) \text { (ont eu des } \\
\text { enfants dans la trentaine, ont eu des } \\
\text { unions antérieures) } \\
\text { - Pères absents ou peu impliqués } \\
\text { avant la naissance* }(3 / 6) \\
\text { - Enfants uniques }(6 / 9) \\
\text { - Seulement } 4 / 9 \text { vivent avec leurs } \\
\text { deux parents pendant toute la } \\
\text { période } 0-5 \text { ans, dont une situation } \\
\text { très conflictuelle } \\
\text { * Souvent, les parents ne vivent } \\
\text { pas en couple ou encore ont des } \\
\text { problèmes de couple. } \\
\text { Chez les } 2 \text { garçons pour lesquels des } \\
\text { antécédents sont notés: } \\
\text { - La mère a eu une enfance } \\
\text { difficile - violence, maladie mentale } \\
\text { des parents }\end{array}$ & $\begin{array}{l}\text { Pour } 3 / 9 \text { jeunes, on n'apprend rien } \\
\text { de spécial ou de nouveau durant cette } \\
\text { période. } \\
\text { - } 2 \text { nouvelles séparations, ce qui porte } \\
\text { à seulement } 2 / 9 \text { enfants vivant en } \\
\text { famille biparentale intacte* } \\
\text { - } 2 / 6 \text { vivent la naissance d'un frère } \\
\text { ou d'une sœur (écart d'âge) } \\
\text { - mesures de soutien aux familles, } \\
\text { généralement sous forme de } \\
\text { services externes et sur mesures } \\
\text { volontaires** (5/9) } \\
\text { * Dans un cas, le jeune perdra } \\
\text { contact avec son père; dans l'autre, } \\
\text { on assistera à un grave conflit post } \\
\text { séparation. } \\
\text { ** Un seul jeune fait l'objet d'un } \\
\text { premier signalement retenu au } \\
\text { cours de cette période et un seul vit } \\
\text { un placement. }\end{array}$ & $\begin{array}{l}\text { - Déficit chronique de l'encadrement" } \\
(9 / 9) \\
\text { - Services externes }(9 / 9) \\
\text { - Placements (9/9) } \\
\text { * Entraîne souvent des conflits par- } \\
\text { ent/enfant, ou beau-parent/enfant, } \\
\text { surtout avec l'adulte qui tente d'im- } \\
\text { poser son autorité. } \\
\text { * Face au dépassement de la mère, un } \\
\text { retour du père est observé dans cer- } \\
\text { tains cas mais sa relation avec le } \\
\text { jeune demeure fragile. } \\
\text { Au niveau de la protection : } \\
\text { - } 5 / 6 \text { filles signalées pour une 1ère } \\
\text { fois (une l'avait été antérieurement) } \\
\text { - } 3 / 3 \text { garçons passent automatique- } \\
\text { ment de L4S à LPJ, sans signalement } \\
\text { Fragilité ou inadaptation des parents } \\
\text { à souligner : } \\
\text { - } 3 \text { mères dépressives, anxieuses, } \\
\text { dont une est aussi suicidaire } \\
\text { ( } 2 / 3 \text { garçons) } \\
1 \text { mère schizophrène } \\
\text { - } 1 \text { père psychotique } \\
\text { - consommation ( } 3 / 9 \text { ) } \\
\text { - impulsivité/violence ( } 2 / 9 \text { ) } \\
\text { - marginalité (1/9) }\end{array}$ \\
\hline
\end{tabular}


Tableau 8. Mauvais traitements chez le Délinquant

\begin{tabular}{|c|c|c|}
\hline $0-5$ ans $(n=9)$ & $6-11$ ans $(n=9)$ & $12-17$ ans $(n=9)$ \\
\hline $\begin{array}{l}\text { Aucune information pour } 6 / 9 \text { jeunes. } \\
\text { On sait peu de choses sur les } 3 \text { autres, } \\
\text { information recueillie a posteriori : } \\
\text { - mauvais traitements } \\
\text { psychologiques de sévérité } \\
\text { inconnue }(2 / 3) \\
\text { - doute d'abus sexuel sérieux de la } \\
\text { part du fils du conjoint de la mère } \\
\text { (1 fille), mais jamais élucidé. }\end{array}$ & $\begin{array}{l}\text { Aucune information sur } 3 / 9 \text { jeunes. } \\
\text { On sait peu de choses sur les } 6 \text { autres, } \\
\text { information recueillie a posteriori dans } \\
5 / 6 \text { cas: } \\
\text { - } 4 / 6 \text { auraient subi des mauvais } \\
\text { traitements psychologiques, } \\
\text { intensité difficile à estimer. } \\
\text { - Parmi ces } 4 \text { jeunes, } 1 \text { fille victime de } \\
\text { négligence assez sévère (conditions } \\
\text { d'existence inadéquates, manque de } \\
\text { supervision et d'encadrement, } \\
\text { parents ne collaborent pas et ne font } \\
\text { pas ce qu'il faut pour régler les } \\
\text { problèmes de l'enfant). } \\
\text { - Cette même fille aurait été victime } \\
\text { d'abus physique de son père avant } \\
\text { l'âge de } 13 \text { ans, mais pas établi } \\
\text { officiellement. } \\
\text { Un autre garçon aurait été investi de } \\
\text { manière fusionnelle par sa mère } \\
\text { entre } 0 \text { et } 12 \text { ans, sans encadrement } \\
\text { cohérent, puis abandonné (mère partie } \\
\text { vivre dans une secte). }\end{array}$ & $\begin{array}{l}\text { 9/9 subissent des mauvais } \\
\text { traitements psychologiques, graves } \\
\text { dans au moins } 3 / 9 \text { cas, mais sévérité } \\
\text { très variable dans l'ensemble: } \\
\text { - Généralement, mère agit cette } \\
\text { violence (violence verbale, rejet, } \\
\text { favoritisme, menaces ou tentatives } \\
\text { de suicide, abandon...) } \\
\text { - quand père ou un beau-père fait } \\
\text { partie de la famille, les deux parents } \\
\text { sont maltraitants (désinvestisse- } \\
\text { ment, rejet, exposition à la violence } \\
\text { conjugale, renversement de rôles, } \\
\text { aliénation parentale...) } \\
\\
\text { 9/9 ne bénéficient pas d'un } \\
\text { encadrement approprié et cohérent: } \\
\text { - impression générale de faibles } \\
\text { capacités parentales et de dépasse- } \\
\text { ment des parents (9/9) } \\
\text { manifestations plus sévères de } \\
\text { manque de supervision et de } \\
\text { négligence présentes dans plus } \\
\text { de la moitié cas (5/9) }\end{array}$ \\
\hline
\end{tabular}


Tableau 9. Problèmes de comportement chez le Délinquant

\begin{tabular}{|c|c|c|}
\hline $0-5$ ans $(n=9)$ & $6-11$ ans $(n=9)$ & $12-17$ ans $(n=9)$ \\
\hline 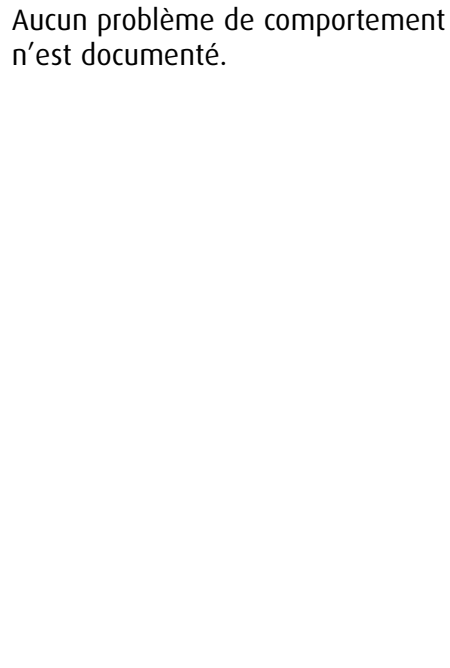 & $\begin{array}{l}\text { Pour } 2 / 9 \text { jeunes, aucun problème de } \\
\text { comportement n'est documenté. } \\
\text { Pour les autres: } \\
\text { - Début de consommation de } \\
\text { psychotropes }(5 / 7) \\
\text { - Problèmes d'adaptation à l'école ou } \\
\text { difficultés académiques ( } 4 / 7) \\
\text { Ces problèmes se poursuivent } \\
\text { généralement à l'adolescence. }\end{array}$ & $\begin{array}{l}\text { - Problèmes de consommation } \\
\text { de substances }(9 / 9) \\
\text { - Fugues, menaces de fugues, } \\
\text { " découchage » }(9 / 9) \\
\text { - Agressivité et violence, verbale ou } \\
\text { physique }(8 / 9) \\
\text { - Opposition, rébellion, refus de } \\
\text { I'autorité, confrontation }(8 / 9) \\
\text { - Propos ou idéations suicidaires, } \\
\text { menaces de suicide }(7 / 9) \\
\text { - Fréquentations de pairs plus âgés, } \\
\text { délinquants ou à risque }(6 / 9) \\
\text { - Vols, fraude, extorsion }(6 / 8) \\
\text { Il convient aussi de souligner que : } \\
\text { - } 3 / 9 \text { jeunes ont commis des } \\
\text { tentatives de suicide } \\
\text { - } 3 / 6 \text { filles sont soupçonnées de } \\
\text { prostitution }\end{array}$ \\
\hline
\end{tabular}

